\title{
A Recoverable Production Planning Model
}

\author{
Juan Pablo Soto Zuluaga \\ Department of Economics and Management, \\ Universitat Pompeu Fabra, \\ R. Trias Fargas 25 - 27, 08005. \\ Barcelona, Spain. \\ juan.soto@econ.upf.es \\ Helena Ramalhinho Lourenço \\ Department of Economics and Management, \\ Universitat Pompeu Fabra, \\ R. Trias Fargas 25 - 27, 08005. \\ Barcelona, Spain. \\ helena.ramalhinho@econ.upf.es
}

July, 2002

\begin{abstract}
Aware of the importance of developing new alternatives to improve the performance of the companies, our purpose in this paper is to develop a medium term production planning model that deals with the concepts of Partnerships and Reverse Logistics. Our model takes advantage of the synergies of integration, developing a model for global production planning that generates the optimal production and purchasing schedule for all the companies integrating a logistic chain. In a second part of the paper we incorporate products returns to the first model proposed, and analyze the implications they have over this model. We use some examples with different configurations of supply chains varying the number of production plants, distribution centers and recovery plants. To solve the model we have combined optimization and simulation procedures.
\end{abstract}

Key words: Reverse Logistics, Production Planning, Remanufacturing, Returns, Supply Chain Management, Simulation, optimization.

JELC: M11,L60,C61.

\section{Introduction}

Nowadays, the logistics condition is best characterized as complex, dynamic and uncertain. Competition is in most markets stronger than ever before, prod- 
uct life cycles have shortened, and business conditions change constantly. Those factors have forced companies to reshape their strategies. Numerous new themes like Global Manufacturing, Third Part Logistics, Partnerships, e-Logistics and Reverse Logistics, are becoming issues that have to be deal with, by all the companies even if they are small or medium sized.

Logistics is officially defined by the Council of Logistics Management as the process of planning, implementing and controlling the efficient and costeffective flow and storage of raw materials, in-process inventory, finished goods and related information from the point of origin to the point of consumption, for the purpose of conforming to customer requirements. More simply, it is the science (and art) of ensuring that the right products reach the right place in the right quantity at the right time and in the right conditions and price, to satisfy customer demand.

Our purpose in this paper is to develop a medium term production planning model dealing with two of these new concepts: Partnerships and Reverse Logistics.

Partnerships remark the benefits of "work together" with our suppliers and distributors. Companies can profit from this integration, improving their competitiveness, through a better estimation of the demand, costs reductions from planning improvements, coordination of activities, etc. Traditional production planning models have been developed, by considering each individual element of a supply chain, and worrying only for individual profits. Our model takes advantage of the synergies of integration developing a global production plan that generates the production and purchasing schedule for all the companies integrating the logistic chain.

On the other hand, Reverse Logistics is a new concept that deals with the management of the products in the reverse way, i.e. it is the process of managing all the flow of returned products and information from the point of consumption to the origin. The actual development of Reverse Logistics strategies in many companies across the world makes it a very interesting topic to work with. It is expected that in few years it will be a crucial element in determining the way in which products will be designed, produced and distributed. The actual legislation of the European Community gives high importance to the recycled products and, in some cases, it has established the responsibility for the end of life products to the manufacturers. Therefore, in the future most of the European companies will have to think about incorporating Reverse Logistics activities in their business operations. Logistics costs are estimated to account for approximately 10.7 percent of the U.S. economy (Rogers, D. and TibbenLembke, R. 1998). However, the exact amount of reverse logistics activity is difficult to determine since most companies do not know how large they are. The study developed by Rogers, D. and Tibben-Lembke,R. (1998), reveals that reverse logistics costs accounted for approximately four percent of the total logistics costs. Applying this mean percentage to U.S. Gross Domestic Product (GDP), they have concluded that reverse logistics costs are estimated to be approximately a 0,5 percent of the total U.S. GDP. Also, based on a respondent sample with 311 reverse logistics managers, they found that reverse logistics 
costs amounted to approximately U\$35 billion in 1997 . The magnitude and impact of reverse logistics varies with industry, channel position and channel choice. However, it is clear that the overall amount of reverse logistics activities in the economy is large and still growing.

We have developed a second model that incorporates returned products to the first model proposed. We analyze the variations and implications of the returns in this model. We use some examples with different configurations of supply chains varying the number of production plants, distribution centers and recovery plants.

The paper is divided into seven sections. Second section starts with a brief introduction to the Reverse Logistics definition. In the third section, we analyze the differences between forward and reverse logistics. We have made also a brief study of the research done in the area. In next section, we developed an aggregated planning model for a multiplant production environment. In section five, we consider the same model but introducing returns with the corresponding remanufacturing processes. In section sixth, we develop a simulation procedure to analyze the performance of the model, and finally some conclusions and future research are stated.

\section{What is Reverse Logistics?}

To have a clear idea about what Reverse Logistics is, let us look at some definitions we have found in the literature:

Krikke, H.(1998), define Reverse Logistics as" the collection, transportation, storage and processing of discarded products".

Fleischmann et al. (1997) says that reverse logistics is "a process which encompasses the logistics activities all the way from used products no longer required by the user to products again usable in a market".

Dowlatshahi, S. (2000) explains Reverse Logistics as "a process in which a manufacturer systematically accepts previously shipped products or parts from the point for consumption for possible recycling, remanufacturing or disposal".

V. Daniel, et al. (2000) says that Reverse Logistics "is the task of recovering discarded products (cores); it may include packaging and shipping materials and back hauling them to a central collection point for either recycling or remanufacturing".

Kroon and Vrijens (1995) says that Reverse Logistics "are the logistic management skills and a activities involved in reducing, managing and disposing of hazardous or non-hazardous waste from packaging and products. It includes reverse distribution, which causes goods and information to flow in the opposite direction from normal logistic activities".

Finally, Rogers, D. and Tibben-Lembke, R. (1998) define Reverse Logistics as "the process of planning, implementing, and controlling the efficient, cost effective flow of raw materials, in - process inventory, finished goods and related information from the point of consumption to the point of origin for the purpose of recapturing value or proper disposal". 
Table 1 shows a brief summary of the elements considered in the definitions.

\begin{tabular}{|c|c|c|c|c|c|}
\hline What is? & Inputs & Activities & Output & From & $T_{0}$ \\
\hline $\begin{array}{l}\text { - Proosss } \\
\text { - Task } \\
\text { - Skills and } \\
\text { Activities }\end{array}$ & $\begin{array}{l}\text { Discarded products. } \\
\text { Used products. } \\
\text { Products or pants } \\
\text { previously shipped. } \\
\text { Prckages and products } \\
\text { from hacarbus and non- } \\
\text { hazardous waste } \\
\text { Irformation } \\
\text { - Raw materials } \\
\text { - In prooss irrertory } \\
\text { - Finished goods } \\
\text { Related Information }\end{array}$ & $\begin{array}{l}\text { - Plarming, } \\
\text { Irqlemerting, } \\
\text { Cortrolling am } \\
\text { efficient and } \\
\text { cost effective } \\
\text { flow. } \\
\text { - Collection } \\
\text { - Transportation } \\
\text { - Storage } \\
\text { - Proossing } \\
\text { - Acceptation } \\
\text { - Recovering } \\
\text { - Packaging } \\
\text { - Shipping } \\
\text { - Reducing } \\
\text { - Maraging } \\
\text { - Disposing }\end{array}$ & $\begin{array}{l}\text { Products again } \\
\text { reusable } \\
\text { - Recycling } \\
\text { Remarnfacturing } \\
\text { - Disposal } \\
\text { - Reducing } \\
\text { - Mamaging } \\
\text { Recapturing vahue }\end{array}$ & $\begin{array}{l}\text { Point of } \\
\text { consumption }\end{array}$ & $\begin{array}{l}\text { Marufacturer } \\
\text { Central } \\
\text { Collection } \\
\text { Point. } \\
\text { Point of } \\
\text { origin }\end{array}$ \\
\hline
\end{tabular}

Figure 1: Table 1: Reverse Logistics definition's elements.

Considering the elements of these definitions we think that the definition given by Rogers, D. and Tibben-Lembke, R. (1998), is the most complete, and incorporates the principal characteristics of what we think reverse Logistics is. So, from now on, for the purpose of our work, when we talk about Reverse Logistics we will consider it according to this definition.

A common problem when talking about reverse logistics, is the confusion existing between reverse and green logistics. The threat that actually exists due to the scarcity and deterioration of natural resources has made companies more conscious about the necessity (obligation, in some countries) of developing green alternatives or ecological ways to do business. Reverse Logistics is commonly misleading with the Green Logistics concept. Redesigning packaging to use less material, or reducing the energy and pollution from transportation are important activities, but they might be better placed in the realm of "green" logistics. If no goods or materials are being sent "backward," the activity probably is not a reverse logistics activity. The confusion remains in the fact that most of the Reverse Logistics activities are lying within the green logistics area.

\section{What is the difference between Forward and Reverse Logistics?}

The research that has been done in reverse logistics is mostly in practitionerrelated journals, rather than academically journals. In fact, there are few articles 
that introduce mathematical or quantitative models. Although it can be seen from the literature that there are many differences between these two concepts that justify the development of different theories in this area. Here we will be concentrated on the operations management issues of reverse logistics, although all the organizational areas may be affected by the introduction of a reverse logistics system into a company. See Rogers and Tibben-Lembke (1998), for a detailed discussion about the incidence of reverse logistics in other areas).

To better analyze the differences between these two concepts we have follow the classification made by Fleischmann et al. (1997), where Reverse Logistics activities are divided into four different research areas: location theory and logistics network design, inventory control and remanufacturing, MRP for recovering and Reverse Logistics and e-commerce.

\subsection{Location theory and logistic network design:}

Apart from the forward logistics network, there are more actors involved in the reverse logistics chain. Here, the supply chain is composed by all the members of the forward logistics network plus third parties, which act as demand points, namely the secondary markets, landfills, charity organizations, and many more. They have a special characteristic, since they do not have a previously established demand, on the contrary, they have limited capacity with respect of different constraints. In the case of landfills for instance, the government regulates the quantity of products that companies can send.

When will a product be returned? Where must it be shipped? Which is the most efficient strategy to choose in order to maximize the firms profits? These are the most common questions that have occupied the researchers in reverse logistics network design.

The high level of uncertainty complicates the design of the reverse logistics network. This uncertainty comes from the fact that the company never knows in advance when, where and how the products will be returned, and the quantities and qualities, may present high variations. Both, quantity and quality of the products returned, are determinants for a suitable network structure since, e.g. high quality products may justify higher transportation costs (and thus a more centralized network structure), whereas extensive transportation of low value products is uneconomical. Moreover, end-markets for recovered products may not be well known, exposing network planning in this context to even more uncertainty (Fleischmann et al.,1997).

In addition, depending of the quantity of products returned it could be necessary to operate with several collection points facilities different from the distribution facilities used in the forward network.

Figure 1, depicts in a very general way the principal components and activities that must be taken into account in the reverse logistics network. We elaborate this figure based on the principal activities of reverse logistics that have been mentioned in the literature (Rogers and Tibben-Lembke, 1998). It is clear that it is not as simple as the Forward one, basically because, when 
the products are collected from the customers, the route they can follow is very different depending on the state of the product. And, considering that not all the product's components are sufficiently valuable to be transported to a manufacturing plant, the problem becomes very complex.

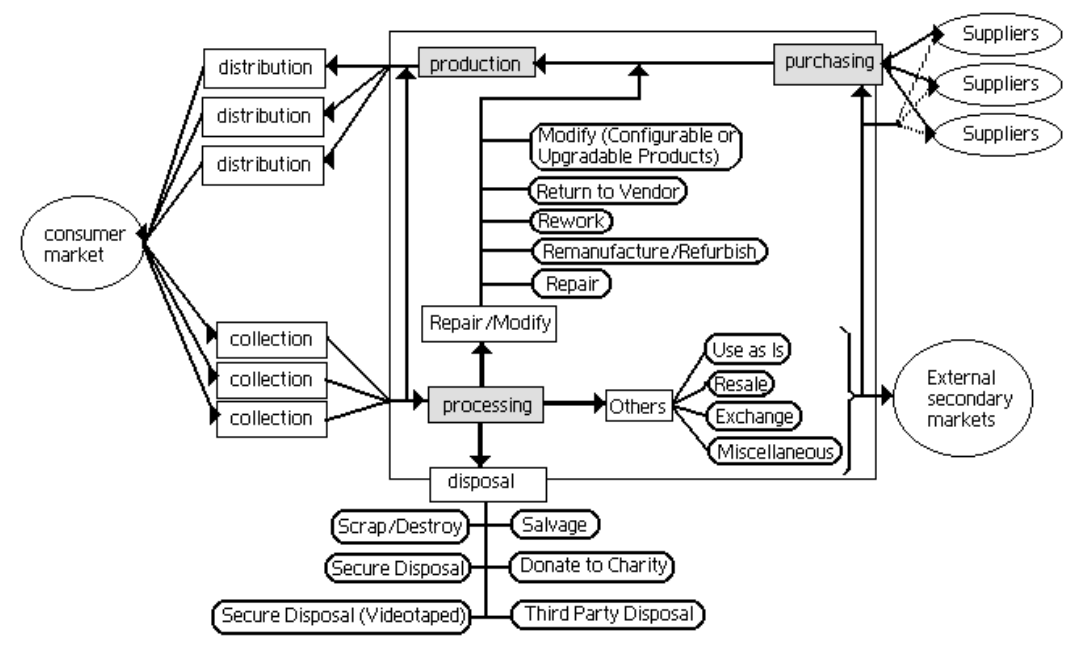

Figure 1: Reverse Logistics Supply Chain

Krikke, H.(1998) states some elements which make the reverse logistics network design different form the forward one:

1. Forward logistics systems are mostly pull systems, while in reverse logistics it is a combination of push and pull since there are clients on both sides of the chain, namely the disposer and the reuser. In forward logistics, only customer markets need to be served and the entire logistic chain, including suppliers (the 'equivalent' of disposers), adjusts itself to it. As a result of the extended producer responsibility, the amount of waste supplied to the reverse logistic system (the push) cannot be influenced in the long run and it has to be matched with demand (the pull). Disposal can serve as an escape route for unwanted waste, but the amount of disposal is limited by legislation, in many countries.

2. Return flows follow a predefined processing graph in which discarded products are transformed into secondary products, components and materials. In forward logistics, this transformation takes place in a production unit, which serves as a source in the network.

3. In reverse logistics, transformation processes tend to be incorporated in the distribution network, covering the entire 'production' process from supply (=disposal) to demand (=reuse). In addition since only a fraction of return flows is valuable, it is likely that in an efficient design, operations are spread over a high number of echelons. Traditional forward logistics models usually focus on one or two echelons. 


\subsection{Inventory control and remanufacturing:}

Within this classification, we include remanufacturing, production planning, inventory control, job scheduling, and all the areas related with production.

A number of factors, including the life-cycle stage of a product and the rate of technological change, influence the quality and quantity of the returns. This characteristic has a marked impact on demand management, and inventory control. The high level of uncertainty arising given the different characteristics in terms of quantity and quality of the returned products, makes more complicated the production planning task, and increases the complexity of the inventory control process.

To understand this complexity, is a good idea to analyze why people return the products. Rogers and Tibben-Lembke, (1998) describe the principal causes for what the people return the products. They found that the most common are described in table 2 which explains the high variety in product quality and quantity:

\begin{tabular}{|l|l|}
\hline Factory repair - Return to vendor for repair & Damaged / Defective \\
\hline Service / Maintenance & Damaged - Cosmetic \\
\hline Agent Order Error - Sales agent ordering error & Dead on Arrival - Did not work \\
\hline Customer Order Error - Ordered wrong material & Defective - Not working correctly \\
\hline Entry Error - System processing error & \\
\hline Shipping Error - Shipped wrong material & Contractual A greements \\
\hline Incomplete Shipment - Ordered items missing & Stock Excess - Too much stock on hand \\
\hline Wrong Quantity & Stock Adjustment - Rotation of stock \\
\hline Duplicate Shipment & Obsolete - Outdated \\
\hline Duplicate Customer Order & Other \\
\hline Not Ordered & Freight Claim - Damaged during shipment \\
\hline Missing Part & Miscellaneous \\
\hline
\end{tabular}

Table 2: Repair and Service codes.

It is important to remark that not only the final customers can return the products, but also retailers and distributors. The process of receive the returned products, imply some different activities of revision and control to determine which is the actual state of the product, and only after that process, it is possible to determine the best strategy to dispose the product, in order to minimize the costs.

Fleischmann et al. (1997) state some differences between reverse and forward logistics inventory control:

- In reverse logistics, as a consequence of the return flow, the inventory level between new component replenishments is no longer necessarily decreasing but may increase also. This loss of monotonicity significantly complicates the underlying mathematical models. A possible starting point for a closer analysis of this aspect, are the cash balancing models comprising in and outbound flows. 
- There are two alternatives for fulfilling the demand that impose an additional set of decisions to be taken. External orders and recovery have to be coordinated.

Daniel Guide, V. et al (2000) determine seven characteristics of the recoverable manufacturing systems that complicate the management, planning, and control of supply chain functions. They are:

- The uncertain timing and quantity of returns

- The need to balance demands with returns

- The need to disassemble the returned products

- The uncertainty in materials recovered from returned items

- The requirement for a reverse logistics network

- The complication of material matching restrictions

- The problems of stochastic routings for materials for repair and remanufacturing operations and highly variable processing times.

As we have mentioned before, it is possible that we must use different parts from different returned products to produce a specific product during the remanufacturing process, and also to mix them with new parts. This also complicates the production process.

Table 3 from Daniel Guide, V. et al (2000), shows a comparison between manufacturing and remanufacturing environment, and the impact it has over the functional areas within an organization.

\subsection{MRP for Recovery:}

Traditional MRP-systems are not feasible for recovery situations for several reasons. One of the main problems is the mismatch of supply and demand, due to the simultaneous release of 'wanted' and 'unwanted' components in the disassembly of returned products. A second major problem is the trade-off between reusing return components or outside procurement .

In Remanufacturing, new products are manufactured using three kinds of components:

- Components that are always retrieved from return products (the quantity is unknown)

- Components that are always purchased new

- Components that can either be purchased new or retrieved from return products, depending on availability and costs. (Krikke, H., 1998)

Fleischmann et al. (1997) states the following arguments, the repair operations needed to convert a returned product back to an 'as new' state depend on the actual condition of the product. This may vary from instance to instance and can in general only be decided after a number of testing and disassembly operations. Therefore, in contrast with traditional manufacturing no well-determined sequence of production steps exists in remanufacturing. This expose planning in a remanufacturing environment to a much higher uncertainty.

A high level of coordination is required in remanufacturing due to the interdependence between different parts and subassemblies. Disassembly of a returned product is not a procurement source for one part but releases various 


\begin{tabular}{|c|c|c|}
\hline Factors & $\begin{array}{c}\text { Recoverable manufacturing } \\
\text { environment }\end{array}$ & $\begin{array}{c}\text { Traditional manufacturing } \\
\text { environment }\end{array}$ \\
\hline Environmental focus & $\begin{array}{l}\text { Seeks to prevent postproduction } \\
\text { waste }\end{array}$ & $\begin{array}{l}\text { Environmentally conscious design } \\
\text { and manufaduring, focus on } \\
\text { Preproduction } \\
\text { Pollution prevention and } \\
\text { remediation }\end{array}$ \\
\hline Logistics & $\begin{array}{l}\text { Fonuand and reverse flouss } \\
\text { Uncertainty in timing and } \\
\text { quantity of retums } \\
\text { Supply-driven flows }\end{array}$ & $\begin{array}{l}\text { Open fonmand flow } \\
\text { No retums } \\
\text { Dem and-driven flous }\end{array}$ \\
\hline $\begin{array}{l}\text { Produdion planning } \\
\text { and control }\end{array}$ & $\begin{array}{l}\text { Need to balance demands uitt } \\
\text { Returns } \\
\text { Material recoveny uncertainty } \\
\text { Stochastic routings and } \\
\text { processing times } \\
\text { Manufacturing system has three } \\
\text { major component: disassembly. } \\
\text { remanufacturing, and } \\
\text { reassembly }\end{array}$ & $\begin{array}{l}\text { No such need } \\
\text { Certaintyin planned materials } \\
\text { Fixed routings and more stable } \\
\text { processing times } \\
\text { Manufacturing system has troo } \\
\text { major components: fabrication } \\
\text { and assembly }\end{array}$ \\
\hline Forecasting & $\begin{array}{l}\text { Forecast both core availability and } \\
\text { end-produdt demand } \\
\text { Must forecast part requirements } \\
\text { because material recovery rates } \\
\text { are uncertain }\end{array}$ & $\begin{array}{l}\text { Forecast only end produds } \\
\text { No parts forecasting needed }\end{array}$ \\
\hline Purchasing & $\begin{array}{l}\text { Highly uncertain material } \\
\text { requirements due to variable } \\
\text { recoveny rates } \\
\text { Cores and parts and components, } \\
\text { replacement parts, components }\end{array}$ & $\begin{array}{l}\text { Material requirements } \\
\text { Deterministic } \\
\text { Raw materials, new parts, and } \\
\text { Components }\end{array}$ \\
\hline $\begin{array}{l}\text { Inventony control and } \\
\text { Management }\end{array}$ & $\begin{array}{l}\text { Types: cores, remanufactured } \\
\text { parts, newp arts, new and } \\
\text { remanufactured substitute parts, } \\
\text { original equipment } \\
\text { manufacturer parts } \\
\text { Must track and provide } \\
\text { accounting for all part types }\end{array}$ & $\begin{array}{l}\text { Types: ranumaterials, work-in- } \\
\text { process, finished goods } \\
\text { Must track and provide } \\
\text { accounting for work-in-process } \\
\text { and finished goods }\end{array}$ \\
\hline
\end{tabular}

Figure 2: Table 3: Comparison between manufacturing and remanufacturing environment

parts simultaneously. Furthermore capacity problems may arise if several parts require the same repair facility. Analogous problems may be encountered for equipment common to new production and repair.

The dependency between components simultaneously obtainable by disassembly and the choice between multiple supply sources (e.g. different returned products) cannot be handled adequately by a simple level-by-level top down approach as in traditional MRP.

Prior to actually processing returned products the specific forms of reuse have to be decided upon. For complex product structures this involves the selection of an appropriate disassembly level of processing options for the components released, taking into account technical as well as economical considerations. 


\subsection{Reverse Logistics and e-commerce}

The importance of reverse logistics in e-commerce is also a new trend that has been started to be analysed. Kokkinaki et al (1999) consider the role of electronic commerce in reverse logistics. They have identified some reverse logistics tasks within the e-commerce applications. Also, they have identified three prominent e-commerce models for the support of reverse logistics activities. At present, the most popular model for e-commerce for reverse logistics is Electronic Marketplaces, which are used for both new and used products. Then, there are sites that use the Web to offer used parts or remanufactured equipment. Finally, there is also a Web based paradigm that incorporates collection, selection, reuse and redistribution.

Logistics aspects of electronic marketplaces cover a great variety of services including inventory management, virtual warehousing, transportation, scheduling and routing, location identification, set up and operational specifications. To simplify the logistics operations of the electronic marketplace, subcontracting third parties to do some or all of the described logistic functions is a common tactic in many electronic marketplaces.

Important issues have arisen in this field. The products now, can be ordered without the physical knowledge by the client, therefore the rates of returns for these products are very high. Products must be delivered at home, but also in case of return, they must be picked up in the customer house.

Table 4 summarizes their analysis in this aspect.

\begin{tabular}{|l|l|}
\hline E-Commerce Applications & Reverse Logistics Tasks \\
\hline -Marketing & - Advertise ment of avallable used products, \\
& parts or material. \\
& - Notification of used products, parts or \\
& material, currently sought. \\
\hline - Purchasing & - Search for suppliers/c ustomers \\
& - Making purchasing commitments \\
& - Receive information of expected \\
& delivery \\
& - Respond to request for sought \\
& used products, parts or materials \\
\hline - Sales & - Price setting (i.e. fixed, negotiations, \\
& auction) \\
& - Order processing \\
& - Tracking and tracing orders \\
& - Customer invoicing, collection and payment \\
\hline Post Salesi Service & - Product tracking \\
& - Customer support \\
& - Customer i product monitoring \\
\hline
\end{tabular}

Table 4: Reverse Logistics activities in e-commerce

There are not much literature developed in this area, but given the importance e-commerce has been acquiring, we have considered important to talk about it independently.

As we can appreciate there are many reasons to develop specific models 
to the reverse logistics activities. The theory developed until today is not very extensive, given the few years that has been dedicated to the study of the reverse logistics.

\section{The multiplant production planning model}

Traditionally, it has been considered that suppliers compete against suppliers, factories against factories, distributors against distributors, and retailers against retailers. This consideration has changed; companies have realized that the competition in the market is not between companies but between supply chains. The final product includes all the inefficiencies and overcosts generated by each one of the companies that compose a supply chain. If the supplier is not efficient, these inefficiency costs are translated to the manufacturer, if again the manufacturer is not efficient, these costs go to the distributor, and finally the product will be not competitive in the market, without caring the distributor and retailer to be efficient. Therefore, the competitiveness of the product must be the result of an excellent management task in all these companies, not only in some of them.

This generates also new challenges for research development. The development of models that consider the demand within the whole supply chain instead of only each individual element, can help companies to improve their global performance. This facilitates reprogramming the production when suppliers are not on time, with the sufficient time to avoid costs of stopping the production, also to program the manufacturing process taking into account the differences in the production costs that exist among factories.

There are basically three different levels of planning in the production environment: the strategic (long term planning) to which belong the business planning; the tactical (medium term planning) which works at an aggregate level of products, examples of this are the Master Production Schedule and the Approximated Capacity Planning, and finally, the Operative Planning (short term planning) with the MRP, job-shop scheduling, etc. In this paper we are working at the tactical level of planning.

The first model proposed is based on the idea to have an integrated production planning within a supply chain of a company with several factories and one central distribution center. The most common models for production planning are focusing in only one plant. We pretend to extend this models to a multiplan production environment to take advantage of coordination and integration of several plants producing the same or similar products. In this way, we will minimize the global production costs of the whole logistics network.

Figure 2 illustrates the supply chain we are considering in this model with multiple plants and one central distribution facility. The plants can be owned by one company, or could be from different partners from a strategic alliance between companies. 


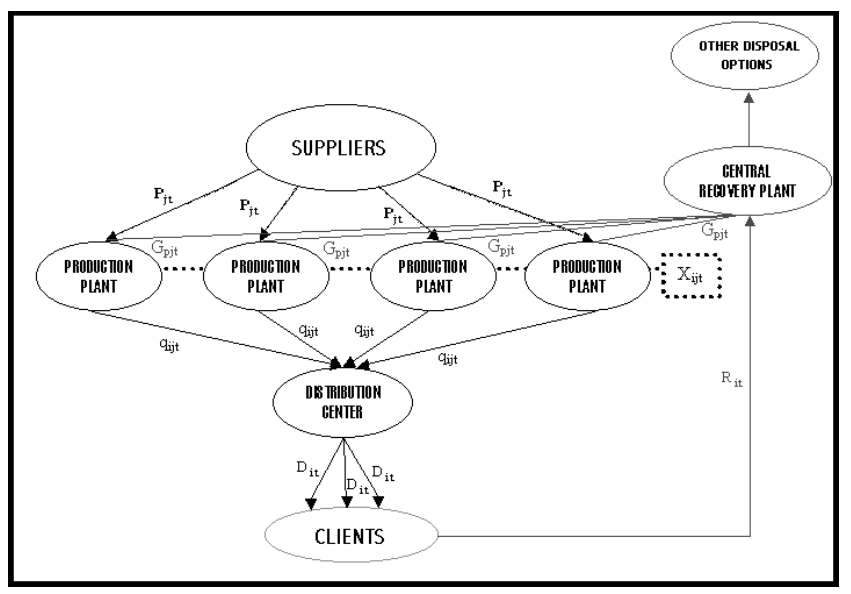

Figure 2: SUPPLY CHAIN

Notice that we have incorporated a Central Recovery Plant, that we will considered later when we introduce returned products to the model.

\section{Model definition}

The Central distribution facility receives the demand for the set of products that the plants manufactured. Each plant can manufactured all or a subset of the product. For each product the bill of materials is knows, i.e. the set of parts and materials that compose each product reference.

The production plants acquire the materials from their suppliers. They must decide the quantity of materials they will purchase at each period, depending on the quantity of products to manufacture, which also must be calculated by the model. Once they have produced their products, they can ship all or part of them to the central distribution center. The manufacturing plants could have inventories of final product and materials. When the central distribution center receive the products, they also prepare and send the products to the consumers following the orders that are represented in the model by the demand. The central distribution center has only inventories on final products, but it is allowed to incur in stockout in case the products are not sufficient to met the period demand. We assume that the demand which is not covered in a period, will be covered in the next period, and also that there are stockout costs derived from the opportunity cost of the sales lost today and in the future.

Let $i=1 \ldots n$, be the index related with the number of manufactured products; $j=1 \ldots m$, the index related to the production plants; $p=1 \ldots P$ the set of materials used; and $t=1 \ldots T$ the planning periods,

We assume the manufacturing plants can use inventories from the previous period and the materials purchased on the current period can be used on that period to produce the articles. Other assumption is that production is done within the period. The periods are usually weeks or biweeks. 
In this model, we assume the demand for each product $i$ on each period $t$ $\left(d_{i t}\right)$ is previously known from the sales forecast of the company, so we only must to decide the quantity of product $i$ to be produced at each factory $j$ at period $t\left(X_{i j t}\right)$, the quantity of product $i$ to be shipped from each factory $j$ to the warehouse at each period $t\left(G_{i j t}\right)$, the quantity of each material $p$ to be purchased at each period $t\left(P_{p t}\right)$ and the inventory units of final products $i$ and materials $p$ to hold at each factory $j$ and at the central distribution center ( $I_{i j t}, M_{j p t}$ respectively).

Each one of the factories has holding and production capacity. We consider also security inventories that must be maintained on each factory. Costs can vary from one plant to another.

We consider the following unit cost:

Total production cost of products $\left(C_{i j t}\right)$,

holding costs of products on the manufacturing plants $\left(H_{i j}\right)$,

transportation costs between the plants and the distribution center $\left(T_{i j t}\right)$,

stockout costs at the distribution center $\left(S_{i t}\right)$,

holding cost at the distribution center $\left(W_{i t}\right)$,

purchase cost of the parts and materials $\left(R_{j p t}\right)$, and

holding costs of the materials $\left(L_{j p t}\right)$.

The objective is to minimize the Total Production Costs of the whole system.

The variables we have used are:

The number of units of product $i$ to produce at plant $j$ at period $t\left(X_{i j t}\right)$, the number of inventory units of product $i$ at plant $j$ at period $t\left(I_{i j t}\right)$,

the number of units of product $i$ to be shipped from plant $j$ to the central distribution center at period $t\left(q_{i j t}\right)$,

the number of units of stockout of product $i$ at central warehouse at period $t\left(Y_{i t}^{-}\right)$,

the number of units to hold of product $i$ at period $t$ at central warehouse $\left(Y_{i t}^{+}\right)$,

the number of units of material $p$ to purchase at plant $j$ at period $t\left(P_{j p t}\right)$, and

the number of units of material $p$ to hold at plant $j$ at period $t\left(M_{j p t}\right)$.

Formally, the objective function is:

$$
\begin{aligned}
& \sum_{j=1}^{m} \sum_{i=1}^{n} \sum_{t=1}^{T} C_{i j t} X_{i j t}+\sum_{j=1}^{m} \sum_{i=1}^{n} \sum_{t=1}^{T} H_{i j} I_{i j t}+\sum_{j=1}^{m} \sum_{i=1}^{n} \sum_{t=1}^{T} T_{i j t} q_{i j t}+\sum_{i=1}^{n} \sum_{t=1}^{T} S_{i t} Y_{i t}^{-} \\
& +\sum_{i=1}^{n} \sum_{t=1}^{T} W_{i t} Y_{i t}^{+}+\sum_{j=1}^{m} \sum_{p=1}^{P} \sum_{t=1}^{T} R_{j p t} P_{j p t}+\sum_{j=1}^{m} \sum_{p=1}^{P} \sum_{t=1}^{T} L_{j p t} M_{j p t}
\end{aligned}
$$

The model has the following constraints:

1. Inventory equation for products: This constraint calculates the inventory level at each period. The inventory of product $i$ at plant $j$ at the end of period $t\left(I_{i j t}\right)$, is equal to the inventory of this product $i$ in this plant $j$ at the 
end of the previous period $t-1 \quad\left(I_{i j(t-1)}\right)$, plus the production quantity of this product $i$ in this plant $j$ during the present period $t\left(X_{i j t}\right)$, minus the quantity of product $i$ shipped from this plant $j$ to the central warehouse at present period $\left(q_{i j t}\right)$.

$$
I_{i j t}=I_{i j(t-1)}+X_{i j t}-q_{i j t} \quad i=1 . . n ; \quad j=1 . . . m ; \quad t=1 \ldots T ;
$$

2. Shipment control: It is necessary to control the quantity of article $i$ shipped from the plant $j$ to the distribution center at each period $t\left(q_{i j t}\right)$. It must be lower or equal than the quantity produced of product $i$ at plant $j$ at this period $t\left(X_{i j t}\right)$, plus the final inventory of product $i$ at plant $j$ at the previous period $t-1\left(I_{i j(t-1)}\right)$.

$$
q_{i j t} \leq X_{i j t}+I_{i j(t-1)} \quad i=1 \ldots n ; \quad j=1 \ldots m ; \quad t=1 \ldots T ;
$$

3. Control of materials: If only can be produced the number of products we can manufacture with the available materials. The quantity of each material $p$ needed to manufacture one unit of product $i$ is defined by $K_{i p}$. For instance, to manufacture product A, are necessary 0 units of material I, 2 units of material II, 1 unit of material III, etc. The total number of units to be produced of product $i$ at plant $j$ at period $t\left(X_{i j t}\right)$, times the number of materials $p$ needed to be produced one unit of product $i\left(K_{i p}\right)$ must be less than or equal to the inventory on material $p$ at plant $j$ at the previous period $t-1\left(M_{j p(t-1)}\right)$ plus the quantity of material $p$ purchased at plant $j$ at period $t\left(P_{j p t}\right)$.

$$
\sum_{i=1}^{n} K_{i p} X_{i j t} \leq M_{j p(t-1)}+P_{j p t} \quad j=1 \ldots m ; t=1 \ldots T ; \quad p=1 \ldots P
$$

4. Inventory capacity for products in factories: The inventory of product $i$ at each factory $j$ at period $t\left(I_{i j t}\right)$ is limited by a security stock quantity defined for each article $i$ and plant $j\left(S S_{i j}\right)$ (could be 0 ), and a Maximum quantity to hold (Holding Capacity) of product $i$ at plant $j\left(B_{i j}\right)$.

$$
S S_{i j} \leq I_{i j t} \leq B_{i j} \quad i=1 \ldots n ; j=1 \ldots m ; \quad t=1 \ldots T ;
$$

5. Inventory equation for materials: To calculate the inventory level of materials on each period. The inventory of material $p$ at plant $j$ at the end of period $t\left(M_{j p t}\right)$, is equal to the inventory of this material $p$ in this plant $j$ at the end of the previous period $(t-1)\left(M_{j p(t-1)}\right)$, plus the purchased quantity of this material $p$ at this plant $j$ during the present period $t\left(P_{j p t}\right)$, minus the quantity of materials $p$ required to manufacture the all products $i$ in period $t$ $\left(\sum_{i=1}^{n} K_{i p} X_{i j t}\right)$. 


$$
M_{j p t}=M_{j p(t-1)}+P_{j p t}-\sum_{i=1}^{n} K_{i p} X_{i j t} \quad j=1 \ldots m ; t=1 \ldots T ; \quad p=1 \ldots P ;
$$

6. Inventory capacity for materials: Each factory $j$ has a maximum holding capacity for material $p\left(V_{j p}\right)$, and a security stock $\left(A_{j p}\right)$. Therefore at each plant $j$, the inventory of material $p$ at period $t\left(M_{j p t}\right)$ is limited by those values.

$$
V_{j p} \leq M_{j p t} \leq A_{j p} \quad j=1 \ldots m ; t=1 \ldots T ; \quad p=1 \ldots P ;
$$

7. Stock out or inventory units at central warehouse: We allow for stock out or inventory units at the central distribution center. They are calculated from the inventory of product $i$ at previous period $(t-1)\left(Y_{i(t-1)}\right)$, plus the total quantity of material $p$ shipped from all the plants $j$ to the central warehouse at period $t\left(q_{i j t}\right)$, minus the demand of product $i$ at period $t\left(d_{i t}\right)$. Notice that if $d_{i t}>Y_{i(t-1)}+\sum_{j=1}^{m} q_{i j t}$, we have units of stockout, and if $d_{i t} \leq$ $Y_{i(t-1)}+\sum_{j=1}^{m} q_{i j t}$, we have inventory units. This means that the variable $Y_{i t}$ could be positive or negative. To solve this situation We divide it into two variables, $Y_{i t}^{+}$and $Y_{i t}^{-}$in such way that if there are units of stock, then $Y_{i t}^{+}=0$ and $Y_{i t}^{-}>0$ and if there are units of stock out then $Y_{i t}^{+} \geq 0$ and $Y_{i t}^{-}=0$. This division allow us to assign different costs to each of the cases and have only variables greater than or equal to 0 .

$$
Y_{i(t-1)}+\sum_{j=1}^{m} q_{i j t}-d_{i t}=Y_{i t} \quad i=1 \ldots n ; \quad t=1 \ldots T
$$

8. Production capacity: This constraint limits the production capacity expressed in hours of production available at each plant. There is a parameter $P T_{i}$ which represent the hours of production needed to manufacture one unit of product $i$, multiplied by the number of units produced of product $i$ at plant $j$ in period $t\left(X_{i j t}\right)$. For each period and each factory, the summation of these multiplication for all the products must be less than or equal to the total production time available at each factory $j$ per period time $t\left(U_{j t}\right)$.

$$
\sum_{i=1}^{n} P T_{i} X_{i j t} \leq U_{j t} \quad j=1 \ldots m ; t=1 \ldots T
$$

Other production capacity constraints can be easily incorporated if necessary. 


\section{9 . Integer and non-negativity constraints.}

All variables are integer and greater than or equal to zero.

In summary the model is the following:

$$
\begin{gathered}
\min \sum_{j=\mathbf{1}}^{m} \sum_{i=1}^{n} \sum_{t=1}^{T} C_{i j t} X_{i j t}+\sum_{j=\mathbf{1}}^{m} \sum_{i=1}^{n} \sum_{t=1}^{T} H_{i j} I_{i j t}+\sum_{j=\mathbf{1}}^{m} \sum_{i=1}^{n} \sum_{t=1}^{T} T_{i j t} q_{i j t}+\sum_{i=\mathbf{1}}^{n} \sum_{t=\mathbf{1}}^{T} S_{i t} Y_{i t}^{-} \\
+\sum_{i=\mathbf{1}}^{n} \sum_{t=1}^{T} W_{i t} Y_{i t}^{+}+\sum_{j=\mathbf{1}}^{m} \sum_{p=\mathbf{1}}^{P} \sum_{t=1}^{T} R_{j p t} P_{j p t}+\sum_{j=\mathbf{1}}^{m} \sum_{p=\mathbf{1}}^{P} \sum_{t=1}^{T} L_{j p t} M_{j p t}
\end{gathered}
$$

Subject to:
1. $I_{i j t}=I_{i j(t-\mathbf{1})}+X_{i j t}-q_{i j t}$

$$
i=1 \ldots n ; \quad j=1 \ldots m ; \quad t=1 \ldots T \text {; }
$$
2. $X_{i j t}+I_{i j(t-1)} \geq q_{i j t}$$$
i=1 \ldots n ; j=1 \ldots m ; \quad t=1 \ldots T \text {; }
$$
3. $\sum_{i=1}^{n} K_{i p} X_{i j t} \leq M_{j p(t-1)}+P_{j p t}$
$j=1 \ldots m ; t=1 \ldots T ; \quad p=1 \ldots P$;
4.S $S S_{i j} \leq I_{i j t} \leq B_{i j}$
$i=1 \ldots n ; \quad j=1 \ldots m ; \quad t=1 \ldots T$;
5. $M_{j p t}=M_{j p(t-1)}+P_{j p t}-\sum_{i=1}^{n} K_{i p} X_{i j t}$
$j=1 . . m ; t=1 . . T ; \quad p=1 \ldots P ;$
$6 . V_{j p} \leq M_{j p t} \leq A_{j p}$
$j=1 \ldots m ; t=1 \ldots T ; \quad p=1 \ldots P ;$
7. $Y_{i(t-1)}+\sum_{j=1}^{m} q_{i j t}-d_{i t}=Y_{i t}$
$i=1 \ldots n ; \quad t=1 \ldots T$;
8. $\sum_{i=1}^{n} P T_{i} X_{i j t} \leq U_{j t}$
$j=1 \ldots m ; t=1 \ldots T$;

9. $X_{i j t} \geq 0$ and integer;

10. $q_{i j t} \geq 0$ and integer;

11. $P_{p t} \geq 0$ and integer;

12. $I_{i j t} \geq 0$ and integer;

$13 . Y_{i t}^{+} \geq 0$ and integer;

14. $Y_{i t}^{-} \geq 0$ and integer;

15. $M_{j p t} \geq 0$ and integer.

\section{Parameters:}

$n=$ Number of articles.

$m=$ Number of plants.

$T=$ Number of periods of time to planning.

$C_{i j t}=$ Production cost of article $i$ at plant $j$ at period $t$

$H_{i j}=$ Holding cost per unit of article $i$ at plant $j$.

$S_{i t}=$ stock out cost per unit of article $i$ at period $t$.

$R_{\text {jpt }}=$ Cost of purchase one unit of material $p$ at plant $j$ at period $t$.

$L_{j p t}=$ Holding cost per unit of material $p$ at plant $j$ at period $t$.

$S S_{i j}=$ Security stock of product $i$ at plant $j$.

$B_{i j}=$ Maximum allowed stock of product $i$ at plant $j$.

$K_{i p}=$ Quantity of material $p$ needed to manufacture one unit of product $i$. 
$A_{j p}=$ Maximum allowed stock of material $p$ at plant $j$.

$V_{j p}=$ Security stock of material $p$ at plant $j$.

$T_{i j t}=$ Transportation cost per unit of product $i$ shipped from plant $j$ to central facility on period $t$.

$d_{i t}=$ Demand of product $i$ at period $t$.

$W_{i t}=$ Holding cost of product $i$ at period $t$ on central warehouse.

$P T_{i}=$ Production time of one unit of product $i$.

$U_{j t}=$ Hours of production capacity in plant $j$ at period $t$.

\section{Variables:}

$X_{i j t}=$ Number of units of product $i$ to produce in plant $j$ on period $t$.

$P_{j p t}=$ Number of units of material $p$ to purchase in plant $j$ at the begining of period $t$.

$q_{i j t}=$ Quantity of product $i$ shipped to central facility from plant $j$ at period $t$.

$I_{i j t}=$ Inventory units of product $i$ in plant $j$ at the end of period $t$.

$M_{j p t}=$ Inventory units of material $p$ in plant $j$ at the end of period $t$.

$Y_{i t}^{-}=$Number of units of stock out of article $i$ at period $t$ at central warehouse.

$Y_{i t}^{+}=$Inventory units of product $i$ at period $t$ at central warehouse.

As we will explain in section 6.1 we solve this model using Lingo software.

\section{The multiplant production planning model with returns}

There are many ways for a company to establish the returns of the products. In the second model we propose, we assume that returns are made through a centralized facility which has the responsibility of receive, disassembly and ship the products to the different production plants. Some authors have studied the problem of disassembly products within a central recovery plant. Krikke (1998) has made a very good approach to this problem, obtaining optimal disassembly strategies based on the quality of the products and parts, and the economical viability of the different alternatives. He has obtained the optimal disposal strategies for each product and in those products where the best alternative is to disassembly, he has determined the optimal level of disassembly for the product. Those alternatives are very different since the quality of the product is unknown and the economical viability of the disassembly process is not the same for all parts and products.

Our model considers that products are returned to a central recovery facility, where they will be classified depending on their quality. The returned product can be fully disassembled on materials or partially disassembled of what it is called assemblies. Assemblies are composed by various materials and given their quality do not need to be slip on primary materials. Therefore, with some 
probability, with respect of their quality, they will be disassembled and the resulting materials and assemblies are prepared to be shipped to the manufacturing plants, where they will be used to manufacture new products. Figure 3 summarize the reception and classification process.

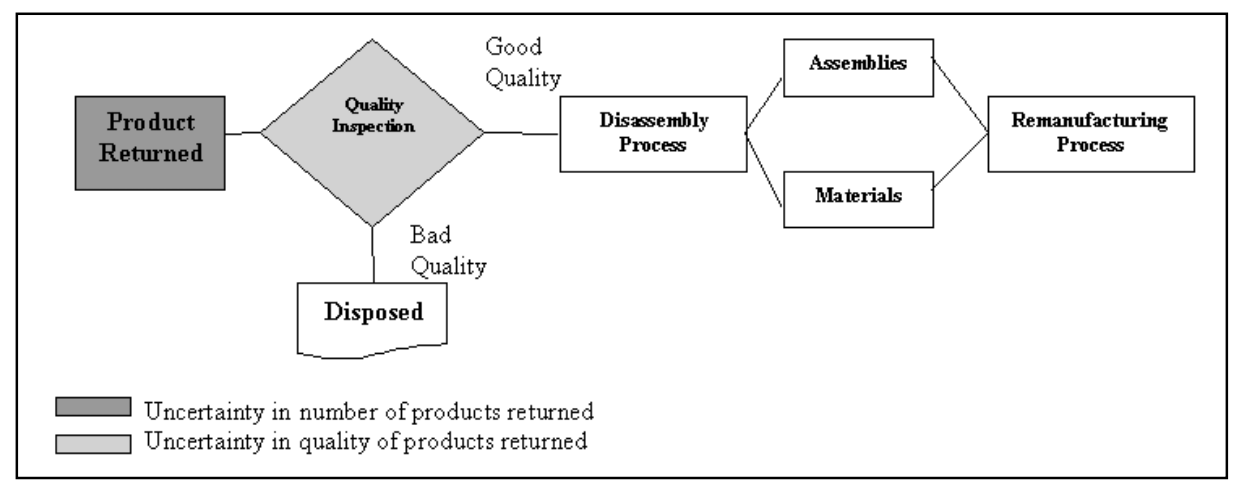

Figure 3: Reception and classification process

The preparation of the products includes all the necessary processes to adequate the materials to be included into the manufacturing process as new materials or assemblies. This implies that, to incorporate these assemblies into the new product, it is necessary to follow a different process than the one used by the factories when working only with new materials. In figure 4 we present a simplification of the bill of materials for product A, defined by Krikke (1998). It illustrates the disassembly tree for this product. In this specific case, product A could be divided into assembly $\mathrm{B}$ and material $\mathrm{E}$ in a first level of disassembly. In a second level of disassembly assembly $\mathrm{B}$ could be divided into material $\mathrm{C}$ and D. Notice that product A could be only disassembled at first level, and material E and assembly B will be the "subproducts" resulting. These "subproducts" will be used as components and materials to manufacture new products.

The introduction of assemblies and remanufacturable materials to the production process complicates the formulation and the size of the problem. We still have the assumptions from the previous model, but we need to add some new ones, to deal with the new remanufacturing environment.

There are many problems that must be considered in order to adequate the model:

A. The uncertainty in the quantity and quality of the products returned affects directly the quantity of materials to purchase and the production time.

B. Different production costs and processes (in an extreme case each unit can follow a different production process).

C. One article can be manufactured with new and reusable parts.

D. In the previous model, it is not necessary to consider in-process inventories. In this model we have to consider them, because now we are introducing 
the assemblies. Therefore, they can go directly to one in-process inventory and increase its quantity.

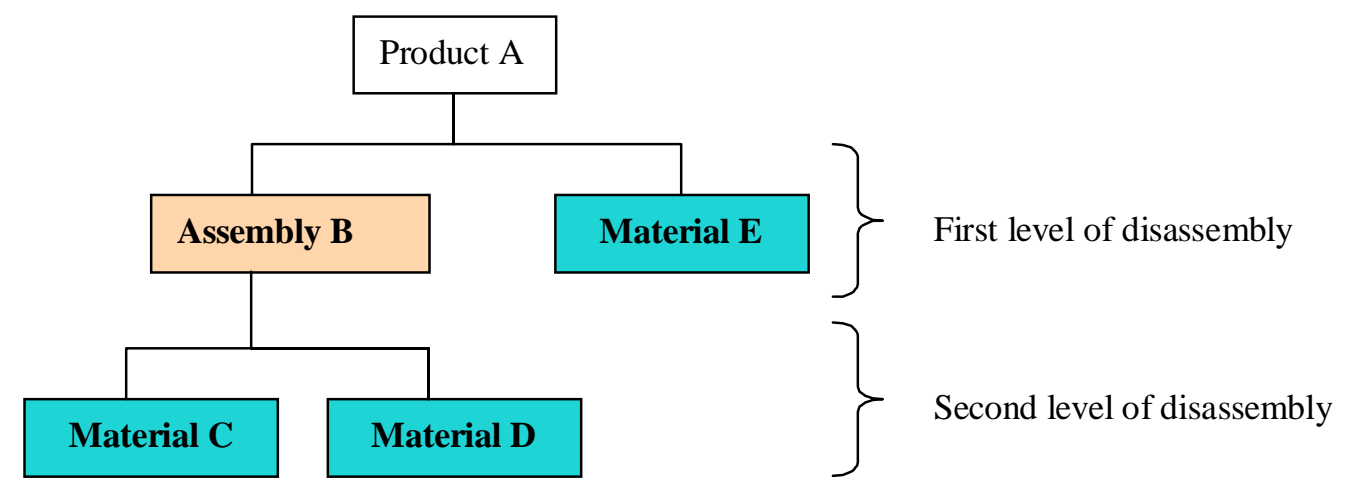

Figure 4: Disassembly tree for product A

To solve these problems, different alternatives were studied. The most simple and effective was to consider the components and reusable materials as new codes of materials, i.e. they are added to the system with new codes, and then different processes are elaborated to each product. Each one of the processes requires a specific type and number of materials, and also has different production costs.

To better understand the problem, let us exemplify it in table 5. It shows an example of the relation between materials and processes for each product. We can observe that product 1 can be manufactured by three different processes. The process 1a, only use new materials, in this case 1 unit of material 1 and 2 units of material 2. This process has a materials cost of 15000 , and a production costs of 7000. The same product (1), can also be manufactured by process $1 \mathrm{~b}$ which use 1 unit of material 2 (which is new), 2 units of reusable material 5, and 1 unit of other reusable material. The materials costs are 13000 and the production costs 6700 . There is also a third process to manufacture product 1 , the process $1 \mathrm{c}$. It use 1 unit of the new material 3 , and a component part (number 98) to manufacture the product, and has also the respective material and production costs.

The model will decide which process should be used to manufacture the product, i.e. how much products will be manufactured, the processes through which they will be produced, the inventories of materials, in-process and final products to be maintained, and the quantity of materials to purchase to the suppliers.

In the formulation of the problem we assume that the cost of the assemblies is the disassembly costs, (which includes also quality inspection and disassembly costs itself) and transportation cost. 


\begin{tabular}{||c|c|c|c|c|c|c|c||}
\cline { 3 - 9 } \multicolumn{2}{c|}{} & \multicolumn{3}{c|}{ Product 1 } & Product 2 & \multicolumn{2}{c||}{ Product 3 } \\
\hline \hline \multicolumn{2}{|c|}{ Materials } & $1 \mathrm{a}$ & $1 \mathrm{~b}$ & $1 \mathrm{c}$ & 2 & $3 \mathrm{a}$ & $3 \mathrm{~b}$ \\
\hline \hline \multirow{3}{*}{ New materials } & 1 & 1 & 0 & 0 & 2 & 1 & 0 \\
& 2 & 2 & 1 & 0 & 5 & 0 & 0 \\
& 3 & 0 & 0 & 1 & 0 & 1 & 0 \\
\hline \hline \multirow{4}{*}{ Reusable materials } & 4 & 0 & 0 & 0 & 0 & 0 & 0 \\
& 5 & 0 & 2 & 0 & 0 & 0 & 1 \\
& 6 & 0 & 0 & 0 & 0 & 0 & 0 \\
& 7 & 0 & 1 & 0 & 0 & 0 & 0 \\
& 8 & 0 & 0 & 0 & 0 & 0 & 0 \\
& $\ldots$ & $\ldots$ & $\ldots$ & $\ldots$ & $\ldots$ & $\ldots$ & $\ldots$ \\
\hline \hline \multirow{4}{*}{ Assemblies } & 98 & 0 & 0 & 1 & 0 & 0 & 0 \\
& 99 & 0 & 0 & 0 & 0 & 0 & 1 \\
& 100 & 0 & 0 & 0 & 0 & 0 & 1 \\
\hline \hline Materials Cost & & 15000 & 13000 & 12500 & 25000 & 14000 & 12500 \\
\hline \hline Production Cost & & 7000 & 6700 & 5500 & 18000 & 9500 & 10000 \\
\hline \hline
\end{tabular}

Table 5: Materials required to produce one unit of products 1,2 and 3 by different production processes.

\subsection{Formulation of the model}

We will now consider an extension of the previous model including the returns. The products are returned to the central recovery facility, where they are revised, classified and organized by the corresponding disposal and remanufacturing strategy. The products which are of good quality for remanufacturing are disassembled and processed until they become materials and assemblies ready to be shipped to the manufacturing plants. The central recovery plant has inventories of reusable materials and assemblies to ship to the factories, and inventories of returned products. The central recovery plant ships the materials to the different factories. The quantity shipped in each period to each factory must be decided by the model, therefore the most profitable alternative will be chosen.

Each factory receives the materials and assemblies from the central recovery facility and uses it to manufacture the products by the different production processes. Let $a=1 \ldots A$, be the set of production processes. Based on the quantity of materials and assemblies obtained from the central recovery plant, the factories chose the quantity of materials to purchase to the suppliers.

The return process at the central recovery plant is affected by several random variables as explained, the number of products returned to the central recovery plant is unknow. Let $F_{i t}$ be the quantity of product $i$ returned on period $t$, we will assume that the average and standard deviation of these variables is known. Let $Q U_{i p}$ be the quality of material or assembly $p$ obtained from the product $i$ which could be good or bad ( 1 or 0 respectively). Let $P R O B_{i p}$, be the probability of one unit of material or assembly $p$ obtained from the disassembly 
process of product $i$ to be of quality $Q U_{i p}$. This probability can take values from 0 to 1 , and we assume it is previously known from different technical specifications. Let $C F_{i p}$ be the number of material or assembly $p$ obtained from the disassembly process of one unit of product $i$, then the average quantity of material $p$ obtained from all products returned at period $t$ is calculated by $\sum_{i=1}^{n} P R O B_{i p}\left[Q U_{i p}\right] * E\left[F_{i t}\right] * C F_{i p}$.

The new model is then formulated like the previous one, but with some variations, namely:

a. The production costs now are differentiated by production processes, so it is calculated in the following form: $\sum_{a=1}^{A} \sum_{j=1}^{m} \sum_{i=1}^{n} \sum_{t=1}^{T} C_{a i j t} X_{a i j t}$, where $C_{a i j t}$ is the production cost of one unit of article $i$ at plant $j$ by process $a$ at period $t$, and $X_{a i j t}$ is the number of units of product $i$ to produce in plant $j$ by process a at period $t$.

b. The transportation cost of material $p$ from central recovery plant to each one of the production plants $j$ at period $t$ is incorporated $\left(G_{j p t}\right)$. This cost is multiplied by the quantity of materials $p$ shipped from central recovery plant to each one of the factories $j$ at period $t\left(O_{j p t}\right)$.

$$
\sum_{j=1}^{m} \sum_{p=1}^{P} \sum_{t=1}^{T} G_{j p t} O_{j p t}
$$

c. The disassembly cost must also be considered. To calculate it is necessary to do the following:

First, it is necessary to calculate the average quantity of material $p$ obtained from all products returned at period $t$ as was explained before. $\left(\sum_{i=1}^{n} P R O B_{i p}\left[Q U_{i p}\right] *\right.$ $\left.E\left[F_{i t}\right] * C F_{i p}\right)$

Second, we multiply this average number of materials returned by the dissassembly cost for material $p$ from product $i$ at period $t$.

Third, we calculate the total for all materials, products and periods.

$$
\sum_{t=1}^{T} \sum_{i=1}^{n} \sum_{p=1}^{P} P R O B_{i p}\left[Q U_{i p}\right] * C F_{i p} * E\left[F_{i t}\right] * D C_{i p t} \text {. }
$$

d. The parameter $K$ is now differentiated also by the production process. This allows us to have the different materials requirements for each process. Notice that the previous production process (from the previous model) is included using only the new materials as in the previous model.

e. We need to separate in the model the new materials and the reusable materials and parts. To do it, we divided the set of materials in two groups, the first group from material 1 to material $n$ which contains only the new materials, and the second group from $(n+1)$ to $P$ which has the reusable materials and the assemblies. It is important to remark that in the model we do not considered directly the materials costs for the reusable materials, because we are calculating it by the sum of quality, disassembly and transportation costs. 
f. It is necessary to consider inventories of returned materials and assemblies at the central recovery plant with the corresponding capacity constraints and holding costs. We define as $I N_{p t}$ the inventory of material or assembly $p$ at period $t$ and with $H O_{p t}$ the correspondent holding cost of material or assembly $p$ at period $t$. The holding capacity for material or assembly $p$ is defined by $M R M_{p}$.

There are also some changes on the restrictions of the model, that will be described next.

1. Inventory equation for reusable materials and assemblies: We called reusable those materials or assemblies obtained from the disassembly process of a returned product. The inventory of material $p$ in period $t\left(I N_{p t}\right)$ is equal to the inventory of this material $p$ at the end of the previous period $(t-1)$ $\left(I N_{p(t-1)}\right)$, plus the estimated returned materials quantity during the present period $t$, minus the quantity of materials $p$ shipped to the plant $j$ at period $t$ $\left(O_{j p t}\right)$.

The estimated returned materials quantity is calculated as explained before for all the reusable materials $(p=(n+1) \ldots P)$ and for all the periods.

$$
\begin{aligned}
I N_{p t} & =I N_{p(t-1)}+\sum_{i=1}^{n}\left(P R O B_{i p}\left[Q U_{i p}\right] * E\left[F_{i t}\right] * C F_{i p}\right)-\sum_{j=1}^{m} O_{j p t} \\
p & =(n+1) \ldots P ; t=1 \ldots T
\end{aligned}
$$

2. Control of shipments: We assume that parts recovered in a period are shipped to the manufacturing plants in the next period. These set of constraints forces the quantity of materials $p$ returned to all the factories $j$ in period $t\left(O_{j p t}\right)$ to be less than or equal to the inventory of materials $p$ at the central recovery plant in the previous period $t-1\left(I N_{p(t-1)}\right)$.

$$
\sum_{j=1}^{m} O_{j p t} \leq I N_{p(t-1)} \quad p=(n+1) \ldots P ; t=1 \ldots T
$$

3. Inventory capacity for reusable materials and assemblies: The inventory of material $p$ at period $t$ in the Central Recovery Plant $\left(I N_{p t}\right)$ is limited by a maximum stock quantity $\left(M R M_{p}\right)$ (holding capacity), and it it must be greater than or equal to 0 . This is calculated for all the reusable materials and for all the periods.

$$
I N_{p t} \leq M R M_{p} \quad p=(n+1) \ldots P ; t=1 \ldots T
$$

4. Inventory equation for materials: Purchased materials are those that manufacturing plants can acquire from external suppliers. The inventory of material $p$ at plant $j$ at the end of period $t\left(M_{j p t}\right)$, is equal to the inventory of 
this material $p$ at this plant $j$ at the end of the previous period $t-1\left(M_{j p(t-1)}\right)$, plus the purchased quantity of this material $p$ at this plant $j$ during the present period $t\left(P_{j p t}\right)$, minus the summation of the number of units to produce by all production processes $a$ of product $i$ at plant $j$ at period $t\left(X_{a i j t}\right)$, times the number of materials $p$ needed to produce one unit of product $i$ by process $a\left(K_{\text {aip }}\right)$ plus the quantity returned of material $p$ to plant $j$ at period $t$ $\left(O_{j p t}\right)$.

$$
\begin{aligned}
M_{j p t} & =M_{j p(t-1)}+P_{j p t}-\left[\sum_{i=1}^{n} \sum_{a=1}^{A}\left(K_{a i p} * X_{a i j t}\right)\right]+O_{j p t} \\
j & =1 \ldots m ; t=1 \ldots T ; p=1 \ldots n ;
\end{aligned}
$$

5. Inventory equation for assemblies: This set of constraints varies from the previous one (with purchased materials) in the case that assemblies cannot be purchased to external suppliers. Therefore the equation is the same but without the purchasing variable $\left(P_{j p t}\right)$.

$$
\begin{aligned}
M_{j p t} & =M_{j p(t-1)}-\left[\sum_{i=1}^{n} \sum_{a=1}^{A}\left(K_{i p a} * X_{a i j t}\right)\right]+O_{j p t} \\
j & =1 \ldots m ; t=1 \ldots T ; p=(n+1) \ldots P
\end{aligned}
$$

6. Inventory capacity for materials: This constraint does not change from the previous model.

$$
V_{j p} \leq M_{j p t} \leq W_{j p} \quad t=1 \ldots T ; \quad p=1 \ldots P ;
$$

7. Control of materials: These constraints ensure that only can be produced the number of products we can produce with the available materials. To do it, we multiply the summation of the number of units to produce by all production processes $a$ of product $i$ at plant $j$ at period $t\left(X_{a i j t}\right)$, times the number of materials $p$ needed to produce one unit of product $i$ by process $a\left(K_{a i p}\right)$. We force this quantity of materials resulting from the previous multiplication to be less than or equal to the inventory of materials $p$ at plant $j$ at the previous period $t-1\left(M_{j p(t-1)}\right)$ plus the quantity of material $p$ purchased at plant $j$ at period $t\left(P_{j p t}\right)$.

$$
\sum_{a=1}^{A} \sum_{i=1}^{n} K_{a i p} X_{a i j t} \leq M_{j p(t-1)}+P_{j p t} \quad j=1 \ldots m ; t=1 \ldots T ; \quad p=1 \ldots P
$$

8. Inventory equation for products: The inventory of product $i$ at plant $j$ at the end of period $t\left(I_{i j t}\right)$, is equal to the inventory of this product $i$ in 
this plant $j$ at the end of the previous period $t-1\left(I_{i j(t-1)}\right)$, plus the production quantity of this product $i$ in this plant $j$ by all the production processes $a$ during the present period $t\left(X_{a i j t}\right)$, minus the quantity of product $i$ shipped from this plant $j$ to the central warehouse at period $t\left(q_{i j t}\right)$.

$$
I_{i j t}=I_{i j(t-1)}+\sum_{a=1}^{A} X_{a i j t}-q_{i j t} \quad i=1 \ldots n ; j=1 \ldots m ; \quad t=1 \ldots T
$$

9. Shipment control: The quantity of product $i$ shipped from the factories $j$ to the distribution center at period $t\left(q_{i j t}\right)$, must be less than or equal to the inventory of product $i$ at plant $j$ at the end of the previous period $(t-$ $1),\left(I_{i j(t-1)}\right)$ plus the quantity produced at each plant $j$ in this period $t$ by all the production processes $a\left(X_{a i j t}\right)$.

$$
\sum_{a=1}^{A} X_{a i j t}+I_{i j(t-1)} \geq q_{i j t} \quad i=1 . . n ; j=1 \ldots m ; \quad t=1 . . T ;
$$

10. Inventory capacity for products: This constraint does not change from the previous model

$$
S S_{i j} \leq I_{i j t} \leq B_{i j} \quad i=1 \ldots n ; j=1 \ldots m ; \quad t=1 \ldots T ;
$$

11. Stockout or Inventory units on central warehouse: This constraint does not change from the previous model

$$
Y_{i(t-1)}+\sum_{j=1}^{m} q_{i j t}-d_{i t}=Y_{i t} \quad i=1 \ldots n ; \quad t=1 \ldots T
$$

12. Production capacity: This constraint limits the production capacity expressed in hours of production available in each plant. There is a parameter $P T_{a i}$ which represent the hours of production needed to manufacture one unit of product $i$ by process $a$, multiplied by the number of units produced of product $i$ by process $a$ at period $t\left(X_{a i j t}\right)$. For each period $t$ and each factory $j$, the summation of these multiplication for all the products $i$ and all the production processes $a$, must be less than or equal to the total production time available in each factory $j$ per period time $t\left(U_{j t}\right)$.

$$
\sum_{a=1}^{A} \sum_{i=1}^{n} P T_{a i} X_{a i j t} \leq U_{j t} \quad j=1 \ldots m ; t=1 \ldots T
$$

Other production capacity constraints can be easily incorporated if necessary. 


\section{Integer and non-negativity constraints.}

All variables are integer and greater than zero.

In summary the new model is the following:

$$
\begin{aligned}
& \min \sum_{a=1}^{A} \sum_{j=1}^{m} \sum_{i=1}^{n} \sum_{t=1}^{T} C_{a i j t} X_{a i j t}+\sum_{j=1}^{m} \sum_{i=1}^{n} \sum_{t=1}^{T} H_{i j} I_{i j t}+\sum_{i=1}^{n} \sum_{t=1}^{T} S_{i t} Y_{i t}^{-}+\sum_{j=1}^{m} \sum_{p=1}^{P} \sum_{t=1}^{T} R_{j p t} P_{j p t} \\
& +\sum_{j=1}^{m} \sum_{p=1}^{P} \sum_{t=1}^{T} L_{j p t} M_{j p t}+\sum_{j=1}^{m} \sum_{i=1}^{n} \sum_{t=1}^{T} T_{i j t} q_{i j t}+\sum_{i=1}^{n} \sum_{t=1}^{T} W_{i t} Y_{i t}^{+}+\sum_{j=1}^{m} \sum_{p=1}^{P} \sum_{t=1}^{T} G_{j p t} O_{j p t} \\
& +\sum_{p=1}^{P} \sum_{t=1}^{T} H O_{p t} I N_{p t}+\sum_{t=1}^{T} \sum_{i=1}^{n} \sum_{p=1}^{P} P R O B_{i p}\left[Q U_{i p}\right] * C F_{i p} * E\left[F_{i t}\right] * D C_{i p t}
\end{aligned}
$$

Subject to:

$$
\begin{aligned}
& 1 . I N_{p t}=I N_{p(t-1)}+\sum_{i=1}^{n}\left(P R O B_{i p}\left[Q U_{i p}\right] * E\left[F_{i t}\right] * C F_{i p}\right)-\sum_{j=1}^{m} O_{j p t} \\
& p=(n+1) \ldots P ; t=1 \ldots T \text {; } \\
& \text { 2. } \sum_{j=1}^{m} O_{j p t} \leq I N_{p(t-1)} \quad p=(n+1) \ldots P ; t=1 \ldots T \text {; } \\
& 3 . I N_{p t} \leq M R M_{p} \quad p=(n+1) \ldots P ; t=1 \ldots T ; \\
& \text { 4. } M_{j p t}=M_{j p(t-1)}+P_{j p t}-\left[\sum_{i=1}^{n} \sum_{a=1}^{A} K_{a i p} X_{a i j t}\right]+O_{j p t} \\
& \text { 5. } M_{j p t}=M_{j p(t-1)}-\left[\sum_{i=1}^{n} \sum_{a=1}^{A}\left(K_{a i p} * X_{a i j t}\right)\right]+O_{j p t} \\
& j=1 \ldots m ; t=1 \ldots T ; p=1 \ldots n ; \\
& \text { 6. } V_{j p} \leq M_{j p t} \leq W_{j p} \\
& j=1 \ldots m ; t=1 \ldots T ; p=(n+1) \ldots P \text {; } \\
& \text { 7. } \sum_{i=1}^{n} \sum_{a=1}^{A} K_{a i p} X_{a i j t} \leq M_{j p(t-1)}+P_{j p t} \\
& \text { 8. } I_{i j t}=I_{i j(t-1)}+\sum_{a=1}^{A} X_{a i j t}-q_{i j t} \\
& t=1 \ldots T ; \quad p=1 \ldots P \text {; } \\
& \text { 9. } \sum^{A} X_{a i j t}+I_{i j(t-1)} \geq q_{i j t} \\
& i=1 \ldots n ; \quad j=1 \ldots m ; \quad t=1 \ldots T ; \\
& \text { 10. } S S_{i j} \leq I_{i j t} \leq B_{i j} \\
& \text { 11. } Y_{i(t-1)}+\sum_{j=1}^{m} q_{i j t}-d_{i t}=Y_{i t} \\
& i=1 \ldots n ; \quad j=1 \ldots m ; \quad t=1 \ldots T ; \\
& i=1 \ldots n ; \quad j=1 \ldots m ; \quad t=1 \ldots T ; \\
& i=1 \ldots n ; \quad t=1 \ldots T \text {; } \\
& \text { 12. } \sum_{a=1}^{A} \sum_{i=1}^{n} P T_{a i} X_{a i j t} \leq U_{j t} \\
& j=1 \ldots m ; t=1 \ldots T \\
& \text { 13. } X_{\text {aijt }} \geq 0 \text { and integer; } \\
& \text { 14. } P_{p t} \geq 0 \text { and integer; } \\
& \text { 15. } I_{i j t} \geq 0 \text { and integer; } \\
& 16 . Y_{i t}^{+} \geq 0 \text { and integer; } \\
& 17 . Y_{i t}^{-} \geq 0 \text { and integer; } \\
& \text { 18. } M_{j p t} \geq 0 \text { and integer; }
\end{aligned}
$$


19. $q_{i j t} \geq 0$ and integer;

20. $O_{j p t} \geq 0$ and integer;

21.IN $N_{p t} \geq 0$ and integer.

\section{Parameters:}

$A=$ Number of production process that has the product with the maximum number of production processes.

$n=$ Number of articles.

$m=$ Number of plants.

$T=$ Number of periods of time to planning.

$C_{\text {aijt }}=$ Production cost of one unit of article $i$ at plant $j$ by process a on period $t$.

$H_{i j}=$ Holding cost per unit of article $i$ at plant $j$.

$S_{i t}=$ stock out cost per unit of article $i$ at period $t$.

$R_{j p t}=$ Cost of purchase one unit of material $p$ at plant $j$ at period $t$.

$L_{j p t}=$ Holding cost per unit of material $p$ at plant $j$ at period $t$.

$S S_{i j}=$ Security stock of product $i$ at plant $j$.

$B_{i j}=$ Maximum allowed stock of product $i$ at plant $j$.

$K_{\text {aip }}=$ Quantity of material $p$ needed to manufacture one unit of product $i$ by process $a$.

$C F_{\text {ip }}=$ Number of units of material $p$ that can be obtained from returned product $i$.

$W_{j p}=$ Maximum allowed stock of material $p$ at plant $j$.

$V_{j p}=$ Security stock of material p at plant $j$.

$T_{i j t}=$ Transportation cost per unit of product $i$ shipped from plant $j$ to central facility on period $t$.

$G_{p j t}=$ Transportation cost per unit of material $p$ shipped from central recovery plant to plant $j$ on period $t$.

$d_{i t}=$ Demand of product $i$ at period $t$.

$W_{i t}=$ Holding cost of product $i$ at period $t$ on central warehouse.

$V_{a i}=$ Production time of one unit of product $i$ by process $a$.

$U_{j t}=$ Hours of production capacity in plant $j$ at period $t$.

$H O_{p t}=$ Holding cost of material $p$ at period $t$ on central recovery plant.

$P R O B_{i p}\left[Q U_{p}\right]=$ Probability of material $p$ resulting from the dissassembly process of product $i$ is of quality $Q U_{p}$ for remanufacturing

$D C_{\text {ipt }}=$ Dissassembly cost for material or assembly $p$ from product $i$ at period $t$.

\section{Variables:}

$X_{a i j t}=$ Number of units of product $i$ to produce in plant $j$ by process a on periodt.

$P_{j p t}=$ Number of units of material $p$ to purchase in plant $j$ at the begining of period $t$.

$q_{i j t}=$ Quantity of product $i$ shipped to central facility from plant $j$ on period $t$. 
$I_{i j t}=$ Inventory units of product $i$ in plant $j$ at the end of period $t$.

$M_{j p t}=$ Inventory units of material $p$ in plant $j$ at the end of period $t$.

$Y_{i t}^{-}=$Number of units of stock out of article $i$ at period $t$ at central warehouse.

$Y_{i t}^{+}=$Inventory units of product $i$ at period $t$ at central warehouse.

$O_{j p t}=$ Quantity of material $p$ shipped from central recovery plant to plant $j$ on period $t$.

$I N_{p t}=$ Inventory units of material $p$ at period $t$ on central recovery plant.

$M R P_{p}=$ Holding capacity of reusable material or assembly $p$ at Central Recovery Plant.

\section{Random Variables}

$E\left[F_{i t}\right]=$ Number of units of product $i$ returned to central recovery plant on period $t$.

$Q U_{i p}=1$ if material $p$ obtained from product $i$, is of good quality for remanufacturing process.

0 if material $p$ obtained from product $i$, is not of good quality for remanufacturing process

\section{Optimization - Simulation Process}

First model is a integer linear programming model and we solve it using the Lingo software. To reduce the computational time, we have using a relaxed model using only the variable $X_{a i j t}$ as integer, but relaxing the remaining integer variables to be real. This model has resulting in a integer optimal solution, therefore it is the optimal solution for the original integer model. With this relaxed model, the computational time was reduced to 3 seconds in average (for the examples we have run).

Dealing with the uncertainty of the variables involved in the second model, in order to solve it, we have combined two different processes. Figure 5 shows the characteristics of the optimization-simulation process (based on Cheung, W. et al (2001)). We have run two different strategies to compare the performance of those strategies.

In first strategy, we ran an optimization model (for the model with returns), taking the expected returns and quality. We obtained the optimal plan. From this optimal plan we decide the number of units to produce, the optimal purchasing strategy and also we obtain an estimative of the inventories and final costs. We assume the company accepts the optimal plan as the production and purchasing strategy.

We then after, consider the optimization results, and run a simulation. In the simulation model, we simulate the quantity of returns for each product, and the quality of the products returned. We use the manufacturing and purchasing schedule produced by the optimization model. Our interest is to analyze the 
inventory behavior for the returned materials and assemblies and the total costs of the model.

As a second strategy, we ran an optimization model as before, but the simulation model only for half of the periods. After that, we use the results to run again the optimization model for the remaining number of periods. In this form the production and purchasing schedule change for the remaining periods. Again, we take the results from this second optimization and use it to run the simulation model for the remaining number of periods.

The inputs and outputs obtained from the optimization and simulation model are summarized in table 6 .

\begin{tabular}{|c|c|}
\hline Optimization & Simulation \\
\hline $\begin{array}{l}\text { Inputs: } \\
\text { Production, holding, stockout and } \\
\text { transportation costs. } \\
\text { Holding and production capacities } \\
\text { Expected number and quality of returns } \\
\text { Demand (from sales forecast) } \\
\text { Initial levels of inventories } \\
\text { Technical factors (Conversion data, } \\
\text { production processes) }\end{array}$ & $\begin{array}{l}\text { Inputs: } \\
\text { Production schedule (from optimization) } \\
\text { Purchasing strategy (from optimization) } \\
\text { Units of products returned (Simulated) } \\
\text { Quality of products returned (Simulated) }\end{array}$ \\
\hline $\begin{array}{l}\text { Outputs: } \\
\text { Optimal production schedule } \\
\text { Optimal purchasing strategy } \\
\text { Estimated inventory levels } \\
\text { Total cost for the planning exercise } \\
\end{array}$ & $\begin{array}{l}\text { Outputs: } \\
\text { Inventory levels } \\
\text { Total costs for the system }\end{array}$ \\
\hline
\end{tabular}

Table 6: Inputs and outputs from optimization and simulation models

We have compared the results from the different optimization-simulation strategies and obtained some conclusions. 


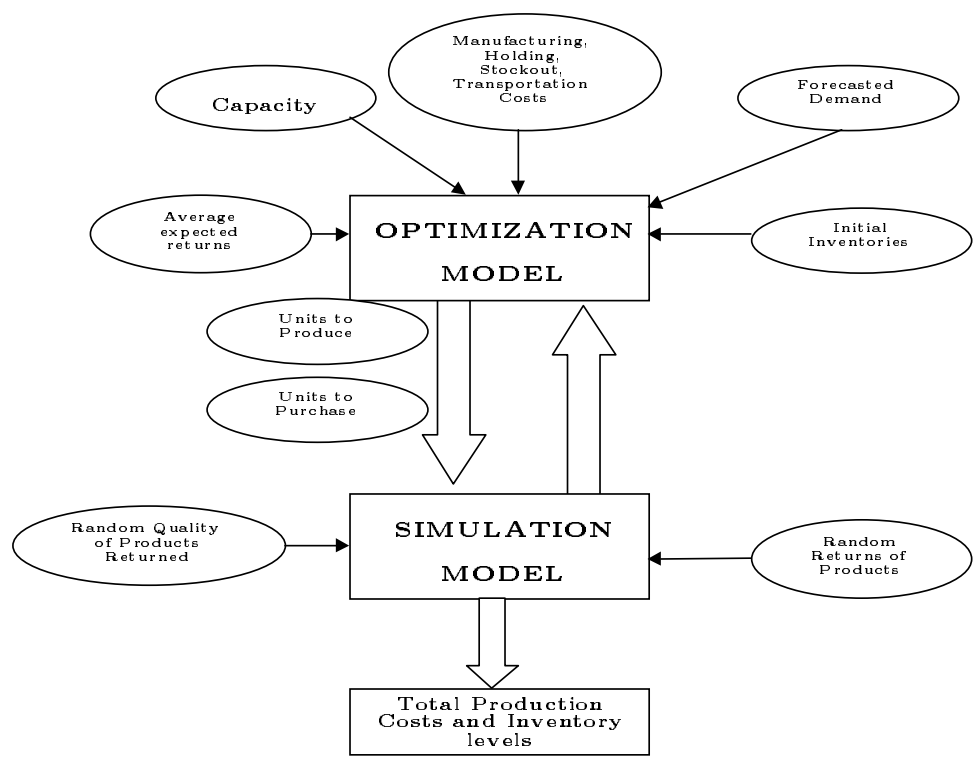

Figure 5: Optimization - Simulation Process

We have considered various examples, varying the number of manufacturing plants, the number of products and the demand of them, the number of materials and the number of production processes arisen from the recovery alternatives.

Figure 6 illustrates the recoverable production system.

The optimization-simulation model evaluates the performance of the results obtained from the optimization model within different scenarios. Later on we would like to use it an iterative process to improve the recoverable production planning in similar way as Cheung, W. et al (2001). The interest of this approach is to compare the possible performance of different scenarios and allow the plan maker to take a better decision. In our example we have established three different scenarios, with a low, medium and high rate of returns. They are defined in terms of the number of returns and quality of products returned.

We are interested in the total costs of the model but, under the three scenarios, we have analyzed also the inventory behavior, since they are a good indicator of the service level of the company. In the next section, we briefly describe some characteristics of both, optimization and simulation model, and we present some results. 


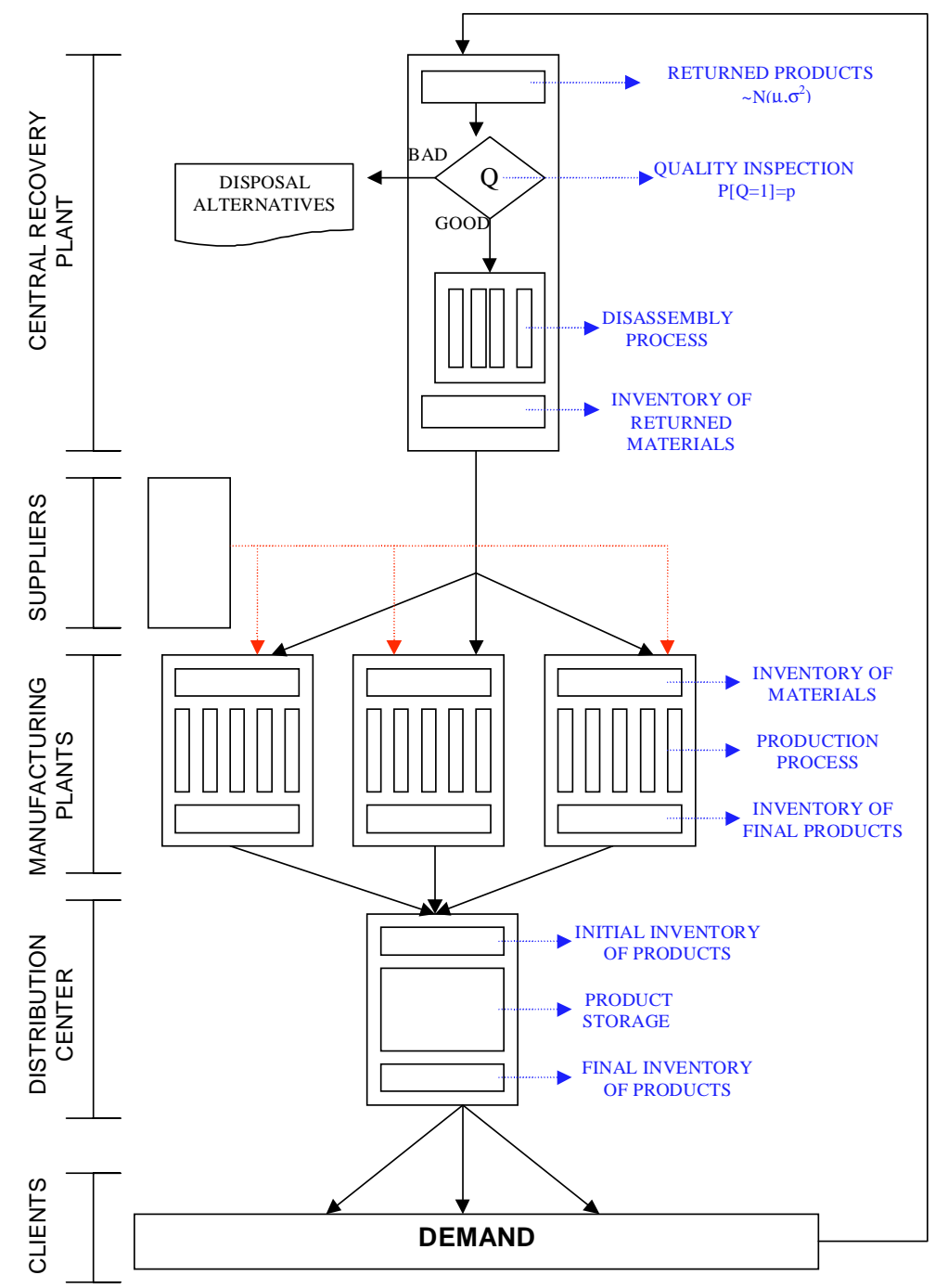

Figure 6: The recoverable production system

\subsection{Optimization Model}

We have used Lingo Software to run the optimization models for the multiplant production planning model with and without returns. To solve it, we have relaxed the integer constraint for all the variables except for the number of units to produce. We have obtained integer optimal solutions for all instances, therefore it is also the optimal solution for the integer problem. The reason for use the relaxation of the model is due the large amount of computational time needed to solve the integer linear programming. For the first instance, Lingo has taken 13 minutes of running time, in a Pentium IV with 1,5 Gghs 
and $256 \mathrm{Mb}$ of RAM memory. But, when we have increased the size of the problem in one additional article or material, the problem becomes too large and the running time increase to more than 60 hours. Therefore, we believe that a heuristic procedure could be useful to obtain results in less time. The optimization model take as parameters all the production costs and an average expected demand per each period, and also an average return and quality of products. The model output is the expected number of units to manufacture and the units of material to purchase at each plant and period. These values are used to make decisions in the quantity of products to be manufactured by each plant and the quantity of materials to be purchased. Once the results have been obtained, we use these results as inputs for the simulation process.

\subsection{Simulation Model}

In order to run the simulation we have developed a program in $\mathrm{C}++$. An additional assumption is made to run correctly the simulation model. When the simulated number of returned products is lower than the ones planned by the optimization model, the program considers an additional purchase order of materials in that period and charge additional costs. This is because in reality companies must buy urgently these materials to meet the planned production scheduling.

The simulation schedule is the following one:

1. Initialization:

a. Set the number of replications to run the simulation.

b. Take the initial inventories and parameters from the optimization model.(Repeat for each replication)

2. For each period:

a. Simulate the number of products returned.

b. Simulate the quality of the products.

c. Calculate production costs and inventory levels for this period.

3. Evaluation:

a. Calculate the average costs and inventories of all the replications.

We assume, that the quantity of returned products, follows a Normal distribution with $\sim N\left(\mu, \sigma^{2}\right)$. Once we have the number generated by the normal distribution, we have used a simple rounding function to obtain the number of products returned. The quality of the products was simulated using a Uniform distribution $(0,1)$ and the technical probabilities used in the optimization model.

\subsection{Results}

We elaborate two different strategies to observe the performace of the model. In the first strategy, we run the optimization model and then use the results to set decisions to run the simulation process. In the second strategy, we run 
the optimization model for all the periods, afterwards we run the simulation process for half of the periods of the example, and then, we run again the optimization process with the data produced by the simulation for the next half of the periods. Once we have obtained the new results from the optimization, we run the simulation for the remaining periods. This last strategy tries to analyse the frequency of running the optimization model.

To run the simulation, we have established three different scenarios for each strategy, depending on the quantity of the returns, and we have observed the performance of the planning scheduling obtained from the optimization process. The first scenario considers a low rate of returns. We assume that returns follow a Normal distribution corresponding to the $5 \%$ of the average expected demand and its standard deviation. The second scenario considers a medium rate of returns, and we define a product return of $15 \%$ of the average expected demand; and the third scenario considers a high level of return, the returns rate is for the $30 \%$ of the average expected demand.

For each strategy, we have ran a total of 5000 replications of the simulation process. The following tables are a summary of the results we have obtained from this process. All the results, are the averages over the 5000 replications of the simulation process.

\subsection{Strategy 1: Optimization with returns + Simulation}

The results from this strategy were obtained from running once the optimization model, and using this results to make the decisions on the simulation process. Table 7 shows the results obtained from this process. We are interested in observing the performance of the reusable materials inventories and the total cost of the system. In both models the demand is known in advance. Also, notice that the results obtained from the optimization is used to set the production scheduling and purchases decision on the simulation model.

In table 7 we present the total costs for the whole process under the following alternatives: optimization with returns, optimization without returns and the three scenarios of the simulation (low, medium and high level of returns). Second and third line, shows the Mean and Standard deviation of the number of returns used at each scenario. The remaining number of lines shows the average number of units of materials and assemblies on inventories under each process. Notice that this value is zero for the optimization without returns, since in this situation the optimal level of inventories is zero. This is different from the optimization with returns, where inventories must be holded because the returned materials can only be shipped at the next period to the factories.

We have considered 2 examples that we will explain next.

\section{Example 1:}


The first example was made based on the following logistic network configuration:

Number of Factories: $\quad 3$

Number of Articles: $\quad 5$

Number of Materials: $\quad 10$

Number of Periods: 12

Number of Production Processes: 5

The results obtained were the following:

\begin{tabular}{|c|c|c|c|c|c|}
\hline \multicolumn{5}{|c|}{ AVERAGE WVENTORIES OF REUSABLE MATERIALS } \\
\hline & 0pt. with Returns & 0pt. W/o Returns & \multicolumn{3}{c|}{ Simulation } \\
\hline Total Cost & $212.210 .700 \mathrm{pta}$ & $234.0000 .800 \mathrm{pta}$ & $397.723 .000 \mathrm{pta}$ & $338.931 .000 \mathrm{pta}$ & $3333.437 .000 \mathrm{pta}$ \\
\hline Mean & & & 1000 & 3000 & 6000 \\
\hline Std. Dev. & & & 356,54 & 1069,61 & 2139,22 \\
\hline Material & Value & Value & low & Medium & high \\
\hline M7 & 6000 & 0 & $-124,0$ & 2800,6 & 9202,5 \\
\hline M8 & 8250 & 0 & $-122,5$ & 9723,2 & 24399,3 \\
\hline M9 & 17637,5 & 0 & 520,0 & 28576,6 & 70427,1 \\
\hline M10 & 37375 & 0 & $-10419,8$ & 26948,2 & 82655,8 \\
\hline
\end{tabular}

Table 7: results from example 1 under strategy 1.

\section{Example 2:}

Second example was made based on the following logisites return network configuration:

Number of Factories: $\quad 2$

Number of Articles: $\quad 3$

Number of Materials: $\quad 15$

Number of Periods: $\quad 12$

Number of Production Processes: 3

The results obtained were the following: 


\begin{tabular}{|c|c|c|c|c|c|}
\hline \multicolumn{5}{|c|}{ AVERAGE NVENTORIES OF REUSABLE MATERIALS } \\
\hline & 0pt. with Returns & 0pt. W/o Returns & \multicolumn{3}{c|}{ Simulation } \\
\hline Total Cost & 216.972 .000 pta & 254.292 .600 pta & $350.820,000$ pta & 322.567 .000 pta & 320.997 .000 pta \\
\hline Mean & & & 600 & 1800 & 3600 \\
\hline Std. Dev. & & & 85,87 & 257,60 & 515,19 \\
\hline Material & Value & Value & low & Medium & high \\
\hline M11 & 98143,6 & 0,0 & 9366,9 & 31004,4 & 63246,8 \\
\hline M12 & 87757,7 & 0,0 & 5161,4 & 21964,4 & 47018,7 \\
\hline M13 & 19455,3 & 0,0 & $-687,8$ & 21388,6 & 54287,1 \\
\hline M14 & 5787,5 & 0,0 & 622,5 & 8968,4 & 21413,1 \\
\hline M15 & 24652,7 & 0,0 & $-7320,6$ & $-4638,1$ & $-640,0$ \\
\hline
\end{tabular}

Table 8: results from example 2 under strategy 1.

In first example the consideration of the returns process into the optimization model, produce better economical results for the company. The reason is the fact that by considering the returns, the plants can use simple and less expensive production processes. It is also important to remark that traditionally the cost of disposal were not consider in the production planning process, therefore the optimization without considering the returns underestimate the real costs of the production process for the company.

In the results from the simulation model, we found that third scenario (high number of returned products) produce better economical results for the company. This is because the stockout costs of the returned materials are very high. When there are stockout of returned materials, it is expected to produce a change in the production plan, because some of these materials cannot be purchased, and therefore the articles must be produced under the traditional production process. Also the company will be forced to purchase new materials urgently at a higher costs.

As expected, the inventories behavior shows high rates of stockout in first scenario, medium in second, and high level of inventories under the third scenario studied.

The results presented in table 8 for second example, are basically the same performance than in the previous example. The optimization with returns provide better results than the optimization without returns. In the simulation, the unique variation was in the scenario with medium level of returns where it was the one with most economical results. The reason is the number of materials in stock was lower than in the scenario with high level of returns, and the stockout costs the number of units in stockout are also lower than in the case of low level of returns. 


\subsection{Strategy 2: Optimization (with returns)- Simulation (half of the Periods) - Optimization - Simulation (re- maining periods)}

Second strategy, consist in running the optimization model, and with this results we simulate the behaviour for half of the total number of periods. Then, we use again the results obtained from the simulation to run the optimization model for the remaining half of periods and with the results we run again the simulation process for those last periods. In table 8 we present the results for this strategy.

\section{Example 1:}

\begin{tabular}{|c|c|c|c|c|c|}
\hline \multicolumn{5}{|c|}{ AVERAGE WVENORIES OF REUSABLE MATERIALS } \\
\hline & 0 pt. with Returns & & \multicolumn{3}{c|}{ Simulation } \\
\hline Total Cost & $212.210 .700 \mathrm{pta}$ & $234.000 .800 \mathrm{pta}$ & $378.470 .000 \mathrm{pta}$ & $296.918 .000 \mathrm{pta}$ & $303.568 .000 \mathrm{pta}$ \\
\hline Mean & & 1000 & 3000 & 6000 \\
\hline Std. Dev. & & 356,54 & 1069,61 & 2139,22 \\
\hline Material & Value & & low & Medium & high \\
\hline M7 & 5716 & & 221,5 & 12902,8 & 21785,2 \\
\hline M8 & 7327 & & $-1976,4$ & 1127,9 & 4180,1 \\
\hline M9 & 15254 & & $-7529,1$ & $-2942,5$ & 2146,1 \\
\hline M10 & 33019 & & $-7220,8$ & 16260,0 & 40768,0 \\
\hline
\end{tabular}

Table 8: results from example 1 under strategy 2.

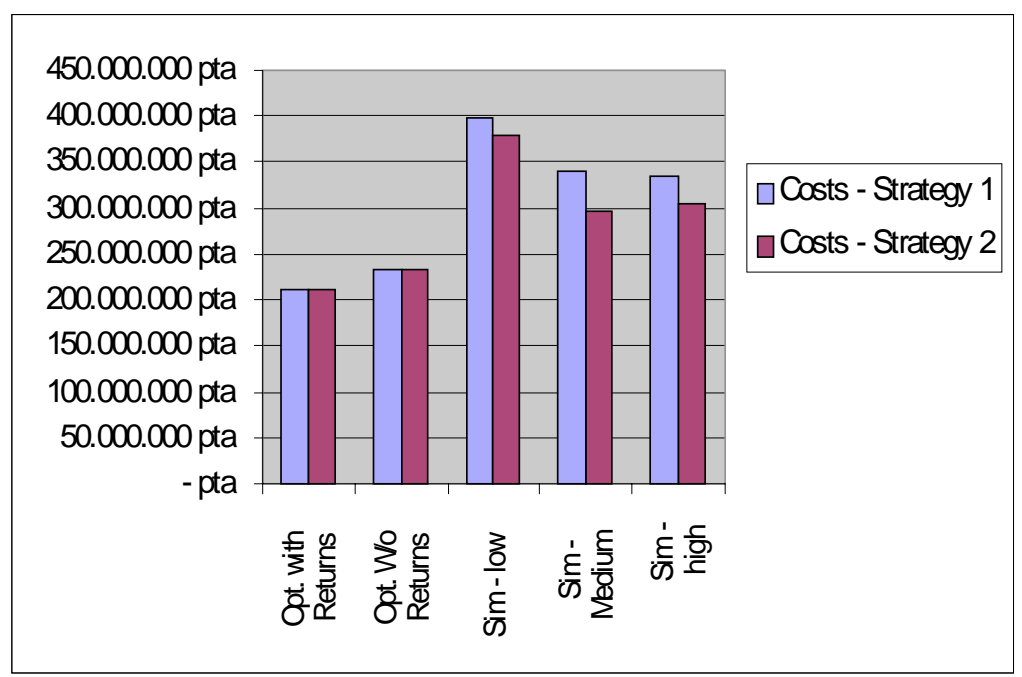

Figure 7: Comparison between total costs under strategies 1 and 2 . 


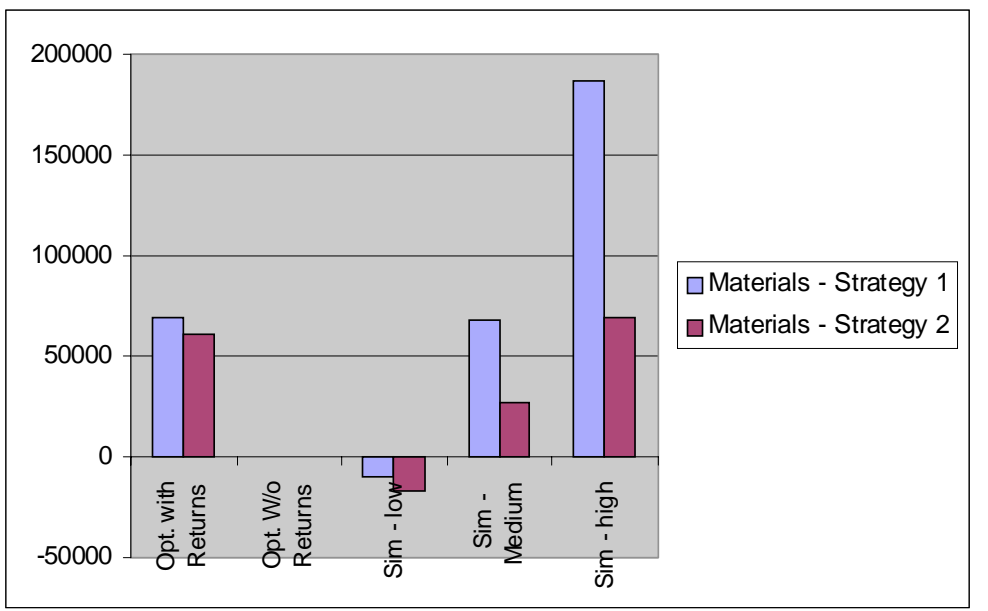

Figure 8: Comparison between inventory levels under strategies 1 and 2 .

Example 2:

\begin{tabular}{|l|c|c|c|c|c|}
\hline \multicolumn{5}{|c|}{ AVERAGE WVNTORIES OF REUSABLE MATERIALS } \\
\hline & 0pt. with Returns & 0pt. W/o Returns & \multicolumn{3}{c|}{ Simulation } \\
\hline Total Cost & $216.972 .000 \mathrm{pta}$ & $254.292 .600 \mathrm{pta}$ & $329.782 .000 \mathrm{pta}$ & $293.580 .000 \mathrm{pta}$ & $284.735 .000 \mathrm{pta}$ \\
\hline Mean & & & 600 & 1800 & 3600 \\
\hline Std. Dev. & & & 85,87 & 257,60 & 515,19 \\
\hline Material & Value & Value & low & Medium & high \\
\hline M11 & 71791,3 & 0,0 & 25192,1 & 40472,9 & 61657,5 \\
\hline M12 & 62669,6 & 0,0 & 17596,6 & 30629,7 & 48470,4 \\
\hline M13 & 19455,3 & 0,0 & $-8028,7$ & $-4244,5$ & 2927,2 \\
\hline M14 & 5787,5 & 0,0 & $-622,1$ & 1954,2 & 5750,5 \\
\hline M15 & 21180,9 & 0,0 & $-2949,5$ & 1415,0 & 9107,7 \\
\hline
\end{tabular}

Table 9: results from example 2 under strategy 2.

Figures 9 and 10 shows the same comparison between strategies one and two for the second example: 


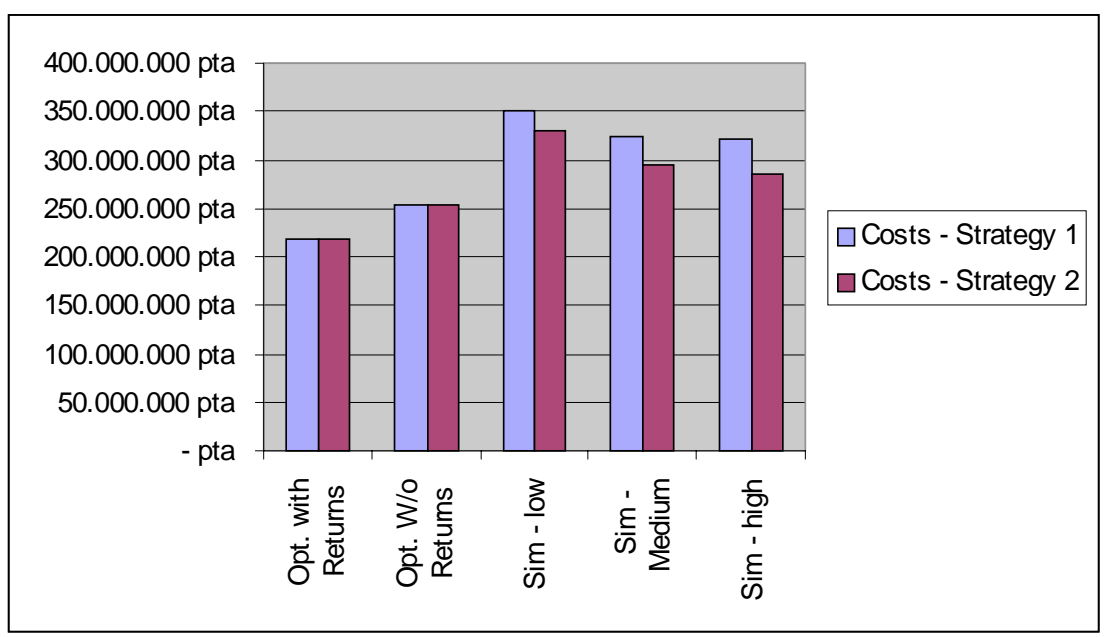

Figure 9: Comparison between total costs under strategies 1 and 2 .

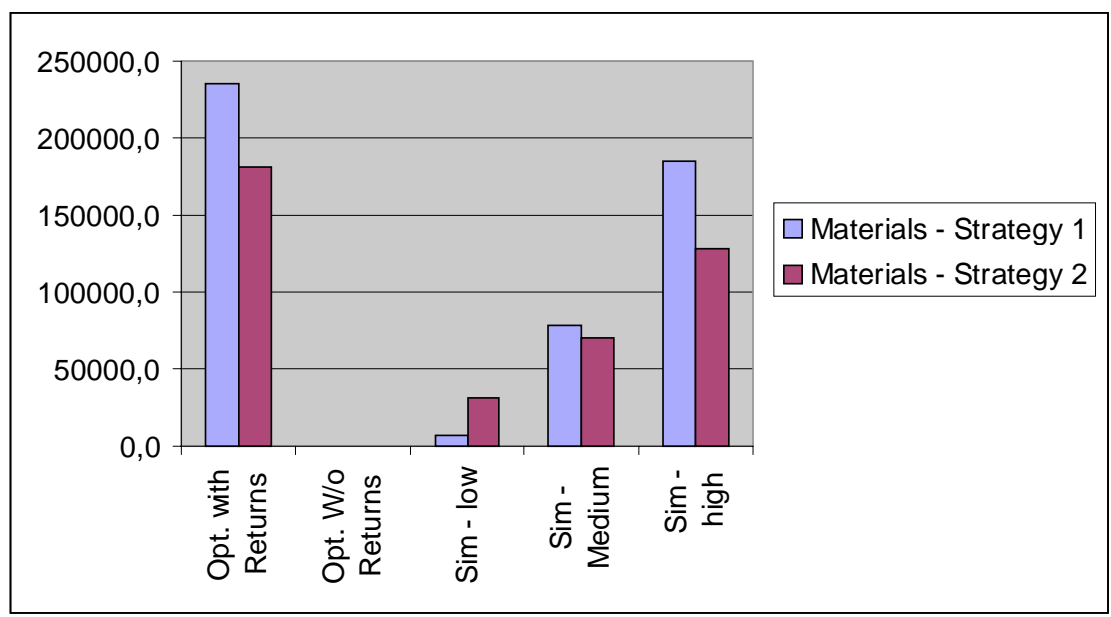

Figure 10: Comparison between inventory levels under strategies 1 and 2.

As expected, in first example, the results obtained by this strategy have improved in comparison with the first strategy. In the same three scenarios of the simulation the total costs have been reduced. Figures 7 and 8, are a comparison between both strategies, in costs and inventory units for this first example

We can appreciate that under the second strategy both, units and costs, have been reduced. Therefore, the use of the simulation process to feedback the optimization process can improve the production schedule and purchasing programming. This leads to the observation that within a Decision Support System, the optimization can be feed directly from real data. The question is how frequent we should run the optimization. The answer depends on the industry. 
Second example, provides the same behavior than the first one, the results from the second strategy presents better results than the ones obtained by first one .

We can appreciate under both examples, that the use of the second strategy can improve the production and purchasing programming. The optimizationsimulation procedure is a good way to improve the decisions made by the company. In the future we plan to extend this model to an iterative process, where the optimization process will be feedback at each period to adapt the results better to the business environment.

\section{Conclusions and directions of future research}

In this work we present two mathematical models for a recoverable production planning system for a production environment within an integrated logistics network.

The first model considers a production plan at the aggregate level for a multiple factories environment. This allows factories to benefit from synergies of an integrated logistics network, obtaining better results for all the partners within the network. The model provides an aggregated production total schedule and also a purchasing planning for each factory during the planning period. The model was formulated as a integer linear programming model and was solved using Lingo Software. The main contribution of this model is the consideration of the integrated logistics network to produce the production planning and purchasing schedule.

In the second model developed, we introduced the returns to the previous production planning model. In this remanufacturing environment, each product can be manufactured by different production processes, depending on the materials used new, reusable or both. The set of production alternatives arising from this different processes, bring to the companies the possibility to choose less costly strategies to manufacture its products. Therefore, the optimization model provides the best combination of manufacturing processes to reduce the total costs for the company.

Given the uncertainty of the model, we have used a combination of optimization and simulation procedures to solve it. We considered two different examples under two different strategies and three different simulation scenarios. In first strategy, we run the optimization model for all the periods and use its results as inputs for the simulation model to obtain finally the total costs of the production process and the inventory performance. In second strategy, we run the optimization model, but the simulation process only for half of the periods. With the results obtained from the simulation model, we feed the optimization model and run it again for the remaining periods, adjusting the purchase and manufacturing schedule. Finally, we run the simulation process for the second half of periods. We have summarized the results from two strategies and obtained some conclusions. The main contribution of this model is the intro- 
duction of the returns to the aggregate planning model, and the utilization of the optimization+simulation procedure to solve it.

From the results we can conclude that the combination of the optimization and simulation procedures can improve the performance of the planning process for the companies. This combination of optimization + simulation give made insights to the decision maker about how to produce a production planning. Also, it give insights on the relationship between the several elements of the production process in terms of the quantity of product on inventory, the production and purchasing schedule, and the total costs of the systems.

The examples used are low size problems, since the optimization + simulation running time becomes too large when the size of the problem increase. For practical purposes and as future research, we plan to develop a heuristic procedure to find a good alternative to solve the optimization model in a reasonable running time. We believe that combination of the heuristic procedure and the simulation, could give us a very good solution for the planning process, more useful for practical purposes.

It would be also interesting to use the optimization+simulation process in a strategy where at each period optimization or heuristic procedures will be feedback by the simulation process, resulting in a plan more accurate to the possible behavior of the products returned.

\section{References}

Rogers, D. and Tibben-Lembke,R. (1998). "Going Backwards: Reverse Logistics Practices and trends". University of Nevada, Reno. Center of Logistics Management. Reverse Logistics Executive Council

Dowlatshahi, S. (2000). "Developing a theory of Reverse Logistics". Interfaces $30: 3$ (143-155).

Fleischmann Moritz, Bloemhof-Ruwaard, Jacqueline M., Van der Laan, Erwin Van Nunen, Jo A.E.E. and Van Wassenhove, Luck N. (1997). "Quantitative models for reverse logistics: A review". European Journal of Operational Research $103(1-17)$.

Kokkinaki, A.I., Dekker, R., Van Nunen, J. and Pappis, (1999). "An exploratory study on Electronic commerce for reverse logistics". Econometric institute report EI-9950/A, Erasmus University of Rotterdam.

Krikke, Harold (1998). "Recovery strategies and reverse logistic network design". Thesis, University of Twente, Enschede, The Netherlands.

Kroon and Vrijens (1995). "Returnable containers: an example of reverse logistics", International Journal of Physical Distribution \& Logistics Management, 25:2, pp 56-68.

V.Daniel R. Guide, Jr., Vaidyanathan Jayaraman, Rajesh Srivastava, and W.C. Benton (2000). "Supply-Chain Management for Recoverable Manufacturing Systems", Interfaces 30:3 (125- 142).

Cheung, W., Leung, L., and Wong, Y.M. (2001). "Strategic Service Network Design for DHL Hong Kong". Interfaces 31:4 (1-14). 